\title{
A SPHERICAL VOLUME INTERACTION DDM APPROACH FOR DIESEL SPRAY MODELING
}

\author{
R. Torelli, ${ }^{1}$ G. D'Errico, $^{1, *}$ T. Lucchini, ${ }^{1}$ V. Ikonomou, ${ }^{2}$ \& R. M. McDavid ${ }^{3}$ \\ ${ }^{1}$ Dipartimento di Energia , Politecnico di Milano, via Lambruschini 4 - 20158 Milano, Italy \\ ${ }^{2}$ Caterpillar UK Engines Company Ltd., Frank Perkins Way, Eastfield, Peterborough PE1 5NA, \\ United Kingdom \\ ${ }^{3}$ Caterpillar Inc, Technical Center - Bldg F, P.O. Box 1875, Peoria IL 61656-1875, United States of \\ America
}

*Address all correspondence to G. D’Errico E-mail: gianluca.derrico@polimi.it

Original Manuscript Submitted: 25/12/2013; Final Draft Received: yy/yy/201y

\begin{abstract}
This work presents an implementation and evaluation of an alternative approach for describing exchange of mass, momentum and energy in Diesel spray CFD simulations using Discrete Droplet Modeling (DDM). During the calculation each parcel in the domain is surrounded by a spherical volume of ambient gas and interacts first with it instead of interacting directly with the cell volume hosting the parcel. In this way the interaction volume is independent of the mesh and can be located in more than one cell. This model was implemented using the OpenFOAM CFD opensource C++ library. It was developed with the aim to reduce grid dependencies related to spray-grid mutual orientation and to the choice of the injector nozzle position with respect to the cell hosting it. All the sub-models constants were set to match experimental data of a chosen baseline case in non-reactant vaporizing conditions. Then the new approach predictions were firstly compared with standard DDM on moving the injector position within the hosting cell and later on varying ambient density and injection pressure of fuel. Also a study of the dependency of the results on the spray-grid mutual orientation was carried out. High-speed imaging and Rayleigh-scattering measurements taken from the ECN web database were used to assess numerical results: a good accuracy in the predictions of liquid and vapor spray penetration as well as axial and radial mixture fraction profiles, can be simultaneously achieved on varying thermo-physical and geometrical settings. If applied to engine calculations, the reduced dependency on the nozzle position becomes appreciable when injector with multiple nozzles are used.
\end{abstract}

KEY WORDS: spray-grid dependency, injector nozzle position, SVI-DDM approach, KHRT breakup model

\section{INTRODUCTION}

Fuel-air mixing, combustion and pollutant formation in Diesel Engines are strictly influenced by how the introduction of the liquid fuel and the interaction of it with the physical domain take place.

Accurate prediction of these processes is fundamental to meet more and more stringent emission regulations and to improve the engine performance.

As it happens for every other physical phenomena, numerical description of Diesel sprays should rely on spatial and temporal averaging and discretization procedures of the relevant differential equations. Different approaches were proposed in the past with the aim to correctly describe the liquid-gas interaction. 
The Discrete Droplet Modeling (DDM) described by Dukowitz (1980) is perhaps the most widely adopted method applied to Diesel spray modeling in the last three decades. This approach consists of a fully-interacting combination of Eulerian fluid and Lagrangian particle calculations and presents at the same time well-known advantages and drawbacks. Among the upsides of this approach, a Lagrangian description of the particles avoids numerical diffusion, and allows individual attributes, such as particle size, composition, etc., to be statistically assigned for each particle. It is also notable that this approach relies on strong basic assumptions such as low liquid volume fraction and homogeneously distributed parcels in the computational cells that are usually not satisfied in the near nozzle region. To partially overcome these issues and to assure numerical stability, it is often necessary to adopt minimum cell sizes larger than the nozzle diameter. This choice may cause an inadequate resolution of the flow structures. Moreover, even when the basic hypothesis are fulfilled, results show strong dependencies on the choice of the grid structure and on the mutual orientation of the spray trajectory with respect to the grid (Abani et al. (2007) and Post et al. (2000)). This makes hard to define a best practice that is independent of the used grid to discretize the physical domain. It becomes even harder in light of the fact that, with the aim to automate more and more CFD calculations, most of the software are lately adopting automatic mesh generation that often produce Cartesian grids. These meshes are mainly characterized by a well organized structure made of hexahedral elements arranged along a Cartesian system of axes. Such a structure is therefore basically independent on the geometry of the physical domain. The present study focuses particularly on the effect that the choice of this grid structure can have if evaluated on varying the injector nozzle position with respect to the cell hosting it.

In DDM approach every parcel injected in the domain represents a statistical entity occupying a precise position at any given time and is characterized by any significant physical quantity considered useful to the analysis. The parcel evolves with time interacting with the surrounding environment that, according to the standard DDM approach, coincides with the cell hosting the parcel in the given time. As briefly outlined before, this approach shows grid dependencies on the mesh structure and size which cannot be neglected. Moreover if the mesh is Cartesian and the injection direction is aligned with one of the main directions of the grid elements, these dependencies are also related to the position of the injector nozzle within the cell hosting it. This study shows that high uncertainties are introduced even only by gradually moving the injector from the center of the cell to one of the vertices. Keeping everything constant but the point from which the liquid is injected, affects the way mass, momentum and energy are transferred from the Lagrangian phase to the Eulerian domain and different liquid length and jet penetration, as well as mixture fraction and velocity distribution have been calculated and pointed out.

In this work an implementation of a different approach based on a spherical volume which acts as intermediary between the Lagrangian and Eulerian phases is proposed. The aim of the paper is to evaluate the application of this approach to Diesel spray modeling by simultaneously comparing it with standard DDM approach and experimental data. These measurements data were taken from the ECN web database (Sandia ECN website, 2013) and include all the parameters listed before.

A sensitivity analysis is finally included to test the robustness of the new approach keeping constant every tunable parameter of the included sub-models (evaporation, turbulence, break-up models, etc.). This analysis was conducted setting every model to match experimental data from a baseline case and then on varying ambient conditions such as density and temperature, as well as using different injection laws (i.e., different injection pressures).

\section{MODEL DESCRIPTION}

\subsection{The VSB2 model as a starting point}

In the past years several authors have implemented different approaches with the aim to reduce the grid dependency that affects the behavior of a liquid spray in engine simulations. The attention was focused on various aspects of the liquid-gas interaction. Abraham and Magi (1999) presented a Virtual Liquid Source (VLS) model that treats the liquid region of the spray as a source of mass, momentum and energy without directly computing the liquid phase. This model uses the assumption that volume and mass occupied by liquid fuel is small relative to to the volume and mass of the total injected fuel. The authors showed that a good agreement between computed and experimental data was achieved but the model was completely inapplicable to simulations of sprays impinging a wall. Schmidt and Rutland (2000) analyzed the grid dependency problem from the point of view of the collision algorithm. In their work they extended the use of the no-time-counter (NTC) method to the general case of varying numbers of droplets in parcels. They outlined that an induced grid dependency appears when a Cartesian 
mesh is used and at the same time a multi-nozzle hollow cone spray is generated. In these conditions it could happen that very close to the injector nozzle there could be a collision interaction between two parcels with totally different trajectories (because injected from different points with different directions). The involved parcels would experience a change in their velocity even if the collision is physically not verified and the main consequence pointed out by the authors was the induction of a non-physical cloverleaf structure of the jet. To overcome this problem they applied the NTC algorithm combined with the use of a polar mesh properly designed for the collision process at each time-step. By doing this they could group the parcels so that they could interact in a more physical way and be more easily sorted when the collision algorithm was applied. Another way to reduce grid dependency is to consider the gaseous interaction volume surrounding each parcel. The idea to consider a sphere around every parcel is not completely new. Kösters and Karlsson (2011) applied it in the implementation of the Stochastic Blob and Bubble spray model, also known as VSB2 model. In that approach the idea was to construct a model that treats the spray and its break-up as one process, instead of summing individual, fragmenting droplets to a spray. VSB2 uses also the definition of an irregular blob that contains droplets sized according to some distribution and replaces the parcel containing identical droplets. The blob then interacts with a gaseous sphere, defined as bubble, whose radius is calculated considering the radius of an equivalent sphere including all the droplets in the parcel. This quantity is finally increased by the turbulent length scale, $l_{t}$, calculated in the cell hosting the parcel according to the well-known definition:

$$
l_{t}=C_{\mu} \frac{k^{\frac{3}{2}}}{\varepsilon}
$$

\subsection{The new model}

What associates the VSB2 model to the Spherical Volume Interaction DDM (SVI-DDM) is the idea to use a spherical volume with which the parcel interacts along its path inside the domain. The first difference between the two methods is that the new model keeps the standard definition of the parcel (i.e., it does not adopt any size distribution) and also does not assign to the sphere a turbulence based radius but a user defined value that in this study is set equal to the minimum cell size. This choice, that will be more clear later, is due to the fact that typical turbulent length scales in Diesel sprays (usually 0.1 to 1.0 times the nozzle diameter) are smaller than the mesh size adopted in this work (five times the nozzle diameter). Another important difference lies in the fact that this method allows interaction with every cells intersected by the sphere and located around the one hosting the parcel (Fig. 1). This guarantees an exchange of quantities between the phases that is in principle independent of the mesh structure. This said, it is clear now that the adoption of a turbulence based radius with a minimum mesh size at least five times bigger than the injector nozzle would almost always result in the generation of spheres that are completely included in the cell where the parcel is located. This would make ineffective the use of the SVI approach with respect to the standard DDM.

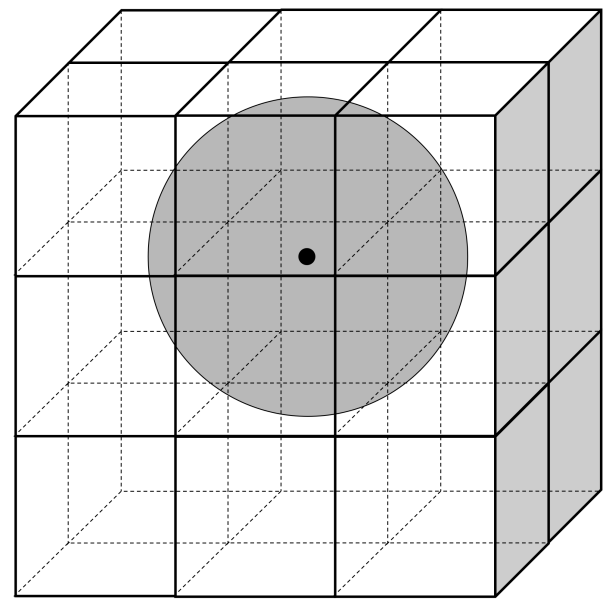

FIG. 1: Interaction volume: the sphere radius is equal to the cell size

An analytical solution is not available to calculate the volumes resulting from the intersections between the sphere and the grid. For this purpose, the choice to use an approximate method was made. Once the sphere is defined, a cloud of $N_{p, t o t}$ uniformly distributed points is collocated inside it. Then they are counted in each of the $k$ cells in the proximity of the parcel and the fraction of the sphere volume included in the $i$-th cell is proportional to ratio of the number of points in the cell, $N_{p, i}$, over the total number of points in the sphere:

$$
V_{i}=V_{s p h} \frac{N_{p, i}}{N_{p, t o t}} \quad, \quad i=1, \ldots, k
$$

The algorithm keeps track of the subdivision of the points in the involved cells and excludes all the ones that are outside of the domain (as it happens when a parcels is very close to the wall boundaries). By doing this, the 
barycenter of every section, $\mathbf{C}_{i}$, can be calculated and this information is then used to interpolate the physical properties of each section. Knowing the interpolated values and by means of weighted averages, it is possible to calculate the overall quantities referred to the gas included in the sphere. For example mass, velocity, temperature and pressure of the gas included in the sphere are computed as follows:

$$
\begin{gathered}
m_{s p h}=\sum_{i=1}^{k} m_{i}=\sum_{i=1}^{k}\left[\tilde{\rho}_{i}\left(\mathbf{C}_{i}\right) V_{i}\right] \\
\mathbf{u}_{s p h}=\frac{\sum_{i=1}^{k} \tilde{\mathbf{u}}_{i}\left(\mathbf{C}_{i}\right) m_{i}}{m_{s p h}} \\
T_{s p h}=\frac{\sum_{i=1}^{k} \tilde{T}_{i}\left(\mathbf{C}_{i}\right) m_{i}}{m_{s p h}} \\
p_{s p h}=\frac{\sum_{i=1}^{k} \tilde{p}_{i}\left(\mathbf{C}_{i}\right) m_{i}}{m_{s p h}}
\end{gathered}
$$

The way the values of the physical quantities at each barycenter are estimated is briefly explained in sec. 2.6.

\subsection{Numerical environment}

The CFD tool used in the present work is OpenFOAM (version 2.0.x), together with the Lib-ICE set of solvers and libraries developed by the authors to simulate I.C. engines (D'Errico et al. (2007) and D'Errico et al. (2012)). Every solver, model, sub-model and library that were not already present in the standard version of OpenFOAM have been implemented in the Lib-ICE including the here presented SVI-DDM approach and a different interpretation of the KHRT breakup sub-model (see Sec. 3.2 and APPENDIX A).

\subsection{Liquid phase equations}

The properties of each parcel (position, velocity, temperature, ...) at any given time are calculated by solving the mass, momentum and energy equations in a Lagrangian way. Multi-component sprays are supported, allowing simulation of realistic fuel surrogates. Droplet momentum is influenced by drag and gravity forces, while the liquid evaporation is estimated through the $D^{2}$-law and by suitable relaxation times calculated under standard and boiling conditions. The energy equation accounts for heat transfer and evaporation. Here, the equations for the liquid phase referred to a droplet of diameter $D$ are presented in summary form; for further details refer to Nordin (2001).

Droplet momentum equation:

$$
m_{d} \frac{d \mathbf{u}_{d}}{d t}=-\frac{\pi D^{2}}{8} \rho C_{d}\left|\mathbf{u}_{d}-\mathbf{u}\right|\left(\mathbf{u}_{d}-\mathbf{u}\right)+m_{d} \mathbf{g}
$$

Droplet mass equation (under standard evaporation):

$$
\frac{d m_{d}}{d t}=-\frac{m_{d}}{\tau_{e}} \quad, \quad \frac{d D}{d t}=-\frac{D}{3 \tau_{e}}
$$

Droplet mass equation (under boiling conditions):

$$
\frac{d m_{d}}{d t}=-\frac{m_{d}}{\tau_{b}} \quad, \quad \frac{d D}{d t}=-\frac{D}{3 \tau_{b}}
$$

Droplet energy equation:

$$
m_{d} \frac{d h_{d}}{d t}=\dot{m}_{d} h_{v}\left(T_{d}\right)+\pi D \kappa \mathrm{Nu}\left(T-T_{d}\right) f
$$

In the equations above, the subscript $d$ refers to the single droplet, $C_{d}$ is the drag coefficient, $\tau_{e}$ and $\tau_{b}$ are respectively the characteristic times for evaporation and boiling conditions. In the last equation $h_{v}$ is the evaporation enthalpy at the temperature of the droplet, $\mathrm{k}$ is the thermal conductivity, $\mathrm{Nu}$ is the Nusselt number and $f$ is a factor which corrects the rate of heat exchange due to presence of mass transfer (see Nordin (2001)).

\subsection{Gas phase equations}

The mass, momentum and energy equations are solved for a compressible, multi-component gas flow using the RANS approach (Nordin (2001), Stiesch (2003)).

Conservation of mass:

$$
\frac{\partial \rho}{\partial t}+\nabla \cdot(\rho \mathbf{U})=\dot{\rho}^{s}
$$

Conservation of species mass fractions: 
$\frac{\partial \rho Y_{i}}{\partial t}+\nabla \cdot\left(\rho \mathbf{U} Y_{i}\right)-\nabla \cdot\left[\left(\mu+\mu_{t}\right) \nabla Y_{i}\right]=\dot{\rho}_{i}^{s}+\dot{\rho}_{i}^{c h e m}$

Conservation of momentum

$$
\begin{array}{r}
\frac{\partial \rho \mathbf{U}}{\partial t}+\nabla \cdot(\rho \mathbf{U U})= \\
-\nabla p+\nabla \cdot\left[\left(\mu+\mu_{t}\right)\left(\nabla \mathbf{U}+(\nabla \mathbf{U})^{T}\right)\right] \\
-\nabla \cdot\left[\left(\mu+\mu_{t}\right)\left(\frac{2}{3} \operatorname{tr}(\nabla \mathbf{U})^{T}\right)\right] \rho \mathbf{g}+\mathbf{F}^{s}
\end{array}
$$

Conservation of energy:

$$
\frac{\partial \rho h}{\partial t}+\nabla \cdot(\rho \mathbf{U} h)-\nabla \cdot\left[\left(\alpha+\alpha_{t}\right) \nabla h\right]=\dot{Q}^{s}+\frac{D p}{d t}
$$

In the equations above $Y_{i}$ represent the mass fraction of the $i$-th specie, $\alpha$ is the thermal diffusivity and all the quantities with the $t$ subscript are contribution obtained from the turbulence model. The symbols $\dot{\rho}^{s}, \dot{\rho}_{i}^{s}, \mathbf{F}^{s}, \dot{Q}^{s}$ identify source terms for mass, momentum and energy exchange between the gas and the liquid phases. The turbulence viscosity, $\mu_{t}$, is provided by the turbulence model that in this work is always the standard k-epsilon as proposed in the original formulation of Launder and Sharma (1974).

Equations (11) to (14) are discretized with the secondorder, finite-volume method on a polyhedral mesh (Jasak (1996), Ferziger and Perik (2002)). The discretization schemes adopted in this work will be shortly presented in sec. 4. Discretization of Laplacian, convection and temporal derivatives terms can be performed with the different schemes originally available in the code (Jasak, 1996). The transient SIMPLE algorithm is used for the pressurevelocity coupling (Issa, 1986).

\subsection{Gas to liquid coupling}

The technique to calculate gas velocity at parcel position slightly differs between the two methods but rely on the same cell-point-face approach (Nordin, 2001). This method lets the parcels experience a continuous velocity field within the computational domain and in both standard and SVI approach it allows a reduction of grid dependency. Firstly, the velocity field, which is stored at the mesh cell centers, is interpolated both at the mesh faces and mesh points locations. Then, a tetrahedron is built with the closest points (cell centers, face centers, mesh points) around the point of interest, $\mathbf{P}$. The distance between $\mathbf{P}$ and one of the tetrahedron faces is $\alpha_{i}$, while $\alpha_{i}+\beta_{i}$ represents the distance between the same face and its opposite vertex. The interpolated velocity at the point $\mathbf{P}$ is:

$$
\mathbf{u}(\mathbf{P})=\sum_{i=1}^{4} \frac{\beta_{i}}{\alpha_{i}+\beta_{i}} \mathbf{u}_{i}
$$

If the standard DDM approach is used, $\mathbf{P}$ represents the parcel position, while in the SVI-DDM $\mathbf{P}$ is the generic barycenter of one of the intersections between the sphere and the mesh. This means that in the new approach proposed here the procedure is repeated for every sphere fraction and then the velocity values are averaged according to the eq. (4). For stability reasons, the same technique cannot be employed to estimate the gas phase temperature and pressure at the parcel positions: to avoid problems in the evaluation of the divergences therms, these quantities are assumed to have the same values of the cell center where the parcel is found ((Nordin, 2001)). As before in the case of the SVI-DDM the cell center values are assigned to the sphere fractions and then averaged according to eqs. (5) and (6).

\subsection{Liquid to gas coupling}

Every parcel is tracked along its path by using a face-toface algorithm. In the standard DDM approach this makes it possible to identify all the cells crossed by each parcel during one time step and to split the Lagrangian source terms of the Eulerian equations accordingly. This technique was proved to increase the accuracy and stability of Diesel spray simulations (Nordin, 2001).

In the SVI-DDM some features are added. The Lagrangian source terms are split according to the cells intersected by the sphere along the parcel path and to the gas mass contained in every sphere section. Since the new methodology is more computational demanding with respect to the standard procedure, the sphere properties are updated within the time step every time the parcel covers a distance larger than the $10 \%$ of the sphere radius. This choice was made after testing the model on a simple case: the percentage of the radius was gradually increased and the choice of the $10 \%$ ensured a good compromise between accuracy and reduction of computational time. 


\section{SPRAY SUB-MODELS}

\subsection{Injection Model}

In accordance with the break-up model (described in sec. 3.2) the Blob-Injection model was used (Stiesch, 2003). Every parcel is injected from a point located in a disk whose size is equal to the injector nozzle: the points are randomly chosen according to a uniform distribution. The frequency of the addition of new parcels is directly related to the fuel injection rate, assuming constant density of the liquid fuel and ideally spherical droplets. Every injected parcel is characterized by the same diameter which is comparable to the size of the nozzle hole on the side of the gas phase. They are introduced in the domain according to the following relation, knowing the total number of parcel to inject:

$$
N(t)=\max \left(1, \Delta t \frac{N_{t o t}}{\left(t_{e o i}-t_{\text {soi }}\right)}\right)
$$

The mass and the velocity assigned to each parcel are calculated as a function of injection profile and total mass to inject. The half-angle of the spray is derived from the assumption that the droplet velocity component perpendicular to the spray axis is proportional to the wave growth rate, $\Omega$, of the most unstable wave of the liquid jet:

$$
\tan \left(\frac{\alpha}{2}\right)=\frac{v_{\perp}}{\left|\mathbf{U}_{p}\right|}=\frac{\Omega \Lambda}{A\left|\mathbf{U}_{p}\right|}=\frac{4 \pi}{A} \sqrt{\frac{\rho_{g}}{\rho_{l}}} f(\mathrm{Ta})
$$

where $\Lambda$ is the wave length and $f(\mathrm{Ta})$ is a function of the Taylor number that asymptotically approaches $\left(3^{0.5} / 6\right)$ for Ta $>100$, which is typically satisfied in Diesel sprays. The quantity $A$ is defined in terms of the length to diameter ratio of the nozzle hole as:

$$
A=3.0+\frac{l_{n o z} / d_{n o z}}{3.6}
$$

The injection direction is then assigned as uniformly distributed in a cone of half-angle $\alpha / 2$.

\subsection{Breakup Model}

Primary breakup is described by means of the BlobInjection model (sec. 3.1) combined with the Wavebreakup model. Secondary break-up is calculated by means of the KHRT model that was used to describe atomization of the spray droplets. Due to a different interpretation of the theory at the base of the KHRT model the implementation here adopted differs from the one presented in the official version of OpenFOAM (Huang and Lipatnikov, 2011), so a complete description of the KHRT is provided in the APPENDIX A.

\subsection{Evaporation, Heat Exchange and Drag Models}

The drag force acting on the droplets is modeled by means of the correlations proposed in Kralj (1995). To correctly describe the mass and energy exchange between the liquid and the surrounding gas, eq. 8 requires expressions for the Sherwood and Nusselt numbers which are modeled according to the approach described in Crowe et al. (1998). According to Kosaka et al. (1995) turbulent dispersion is one of the processes which produce the inhomogeneous distribution of fuel mass concentration, but for the purposes of this work and to reduce the results sensitivity to the turbulence model (Stiesch, 2003) effects of turbulent dispersion were not considered. Collision models were also not used, because of their limited effects on the Sauter Mean Radius (SMR) of an evaporating spray, as it is illustrated in Baumgarten (2006).

\section{MESH MANAGEMENT TOOLS}

The grids used in this work, due to the very simple geometry of the physical domain, were created by means of the blockMesh OpenFOAM utility. What is important to mention is that the adptive local mesh-refinement (ALMR) technique was used as done in Lucchini et al. (2011).

\subsection{Refinement strategy}

To preserve a good quality of the mesh, only hexahedral and degenerated hexahedral cells (wedges) can be refined. An initial computational mesh has to be provided by the user and the size should be fine enough to correctly reproduce the geometrical domain to be simulated and the main details of the initial flow-field (Jasak, 1996). A geometric field is chosen as an error estimator and when its values lie in a user-specified interval the parent cell is split into eight child cells by introducing new nodes at the cell centroid and at the mesh face centers (Jasak (1996)). An arbitrary level of refinement is chosen by the user, and a maximum number of cells can be specified in order to keep a desired value of the mesh size. Refined cells are marked with a flag number and by means of it the mesh can be easily unrefined when the values of the error estimator are outside 
the specified interval. In this work the unrefinement feature was disabled while the initial mesh size was set equal to $4 \mathrm{~mm}$, with three levels of auto-refinement. In this way a minimum mesh size of $0.5 \mathrm{~mm}$ was reached. The choice to adopt a $0.5 \mathrm{~mm}$ size was due to the necessity of keeping a reasonable number of cell during the calculations, especially for what concerns the engine simulations that will be shown in sec. 5.7 and that will involve a number of elements in the range of 2.5-3.0 millions.

\subsection{Refinement criterion}

The geometric field used as a refinement criterion is represented by the total fuel mass fraction (liquid and gas) in each cell:

$$
Y_{l+g}=\frac{m_{f, l}+\rho Y_{t f} V_{c e l l}}{\rho V_{\text {cell }}}
$$

where $m_{f, l}$ is the liquid mass of all the parcels belonging to the cell, $Y_{t f}$ is the fuel mass fraction in the continuous phase, $\rho$ is the gas phase density and $V_{\text {cell }}$ is the cell volume. The lower threshold value was set to $10^{-4}$ while the higher was 1 . This allows an adequate refinement of the mesh close to the nozzle in the first time steps.

\section{RESULTS AND DISCUSSION}

The proposed approach was validated with different sets of experiments run in non-reacting conditions. All the tests were conducted in an optical, constant-volume vessel with a single-component fuel (n-dodecane, $\mathrm{C}_{12} \mathrm{H}_{26}$ ) and the collected data were used to tune the spray model constants. As briefly outlined in the introduction, in order to select a common setup for all the cases, experimental data of a baseline case were used. After reaching a good agreement in terms of liquid length and fuel vapor penetration between calculated results and experimental measurements, four analyses were conducted. In the first one the injector position was moved from the center of the cell (taken as reference condition) to one of the corner vertices, considering also an intermediate position. This was made for both, standard and SVI DDM with the aim to test the predictive capability of the latter. After this first phase, ambient density and injection pressure were varied to test the robustness of the chosen setup and the sensitivity of the new approach to the varied conditions. The third analysis was focused on the dependency of the results on the spray-grid mutual orientation. Finally a simplified engine case was simulated to test the behavior of the new model when multiple-nozzle injectors are used.

\subsection{SANDIA constant volume vessel}

All the simulations in this work were conducted on a mesh representing the SANDIA constant volume vessel. The vessel has a cubical-shaped combustion chamber. The characteristic dimension of the cube is $108 \mathrm{~mm}$. The fuel injector is located in one side port using a metal insert that forms the right wall of the combustion chamber. Two spark plugs and a mixing fan are mounted in another metal insert that forms the top wall of the chamber. Optical access is provided by four sapphire windows with clear apertures of $102 \mathrm{~mm}$ located in the other four ports. For wall heat transfer modeling purposes, the steel vessel and metal inserts for the injector and spark plugs are made of 4340 steel.

In this chamber a mixture of gases can be burnt to reach the necessary conditions in terms of pressure, density, temperature and chemical composition. To obtain a nonreacting environment for the spray to evolve, a stoichiometric mixture was burnt to obtain a negligible oxygen content after the combustion.

To describe the geometry, a cubic mesh whose edge is $108 \mathrm{~mm}$ was created. The grid is three-dimensional and its cells are perfectly cubic with an initial size of $4 \mathrm{~mm}$. The injector is placed in a cell belonging to what will become the second plane of cells starting from the wall after the refinement procedure.

Fig. 2 is a simple scheme to show the three different positions chosen to perform the calculations.

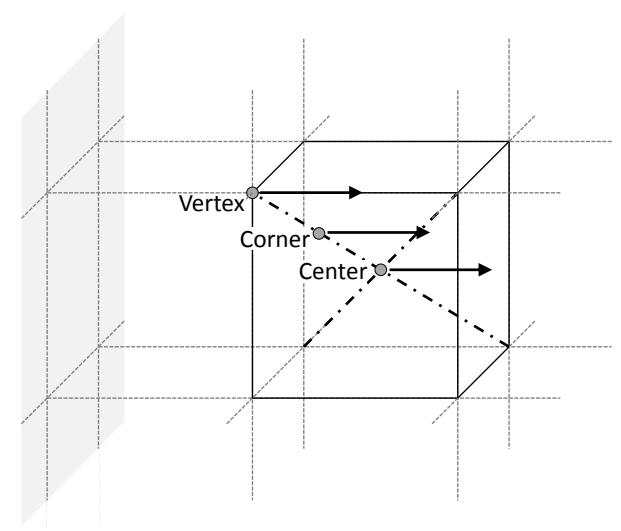

FIG. 2: Injection positions and directions

\subsection{Analysis strategy}

Among the numerous data available from the experiments run on the Sandia Constant Volume Vessel, the Spray A 
series was selected. The Table 1 summarizes the analyzed cases. The first row refers to the baseline case on which the various sub-models were set, second and third row to the ambient density variation the last two to injection pressure variation.

TABLE 1: Simulated cases

\begin{tabular}{|r|c|c|c|c|}
\hline Case Name & $\begin{array}{c}\text { Injection } \\
\text { Pressure } \\
{[\mathrm{MPa}]}\end{array}$ & $\begin{array}{c}\text { Ambient } \\
\text { Density } \\
{\left[\mathrm{kg} / \mathrm{m}^{3}\right]}\end{array}$ & $\begin{array}{c}\text { Ambient } \\
\text { Temperature } \\
{[\mathrm{K}]}\end{array}$ & $\begin{array}{c}\text { Ambient } \\
\text { Pressure } \\
{[\mathrm{MPa}]}\end{array}$ \\
\hline Baseline & 150 & 22.8 & 900 & 6 \\
\hline Density15.2 & 150 & 15.2 & 900 & 4 \\
\hline Density7.6 & 150 & 7.6 & 900 & 2 \\
\hline Pressure100 & 100 & 22.8 & 900 & 6 \\
\hline Pressure50 & 50 & 22.8 & 900 & 6 \\
\hline
\end{tabular}

As said before the reference case had the injector placed in the cell center. The model constants (turbulence, breakup, etc.) were set to match experimental data referring to the baseline case.

The Table 2 reports the constants used to tune the turbulence model. If compared to the original formulation $C_{1}$ is the only constant that was tuned to match experimental data better.

TABLE 2: $k$-epsilon turbulence model constants

\begin{tabular}{|c|c|c|c|c|c|}
\hline$C_{\mu}$ & $C_{1}$ & $C_{2}$ & $C_{3}$ & $\sigma_{k}$ & $\sigma_{\epsilon}$ \\
\hline 0.09 & 1.552 & 1.92 & -0.33 & 1 & 1.4 \\
\hline
\end{tabular}

The graphic in Fig. 3 shows the achieved agreement in terms of liquid length and vapor penetration.

The calculated liquid length was measured taking the distance of the parcel representing the $99 \%$ of liquid mass in system, while the jet penetration is taken as the farthest point along the injection direction where the mixture fraction, $\mathrm{Z}$, is at least equal to the $0.1 \%$ of the cell volume. Fig. 4 shows good results also in terms of Mixture Fraction profiles at different locations and contours of Fig. 5 confirm this. Fig. 5 shows also that the calculated results are not able to catch finer spray structure present in experimental measures. This is mostly due to the nature of RANS simulations which leads to time-averaged fields characterized by an isotropic turbulence combined with what is mainly an axi-symmetrical problem. Furthermore, the choice of a minimum mesh size of $0.5 \mathrm{~mm}$ does not allow to predict smaller structures.

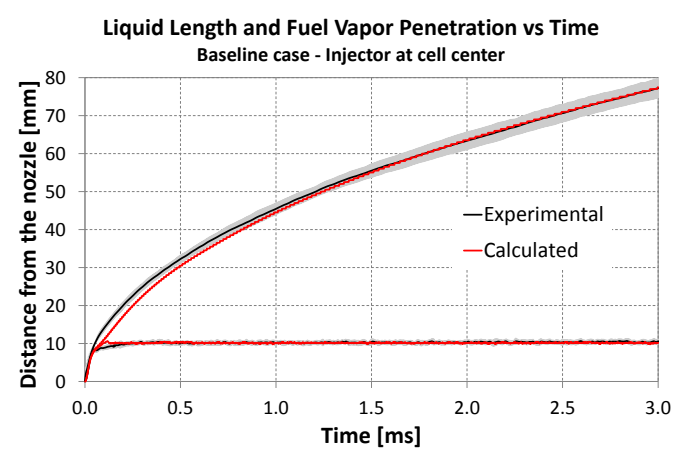

FIG. 3: Liquid Length and Vapor Penetration. Baseline

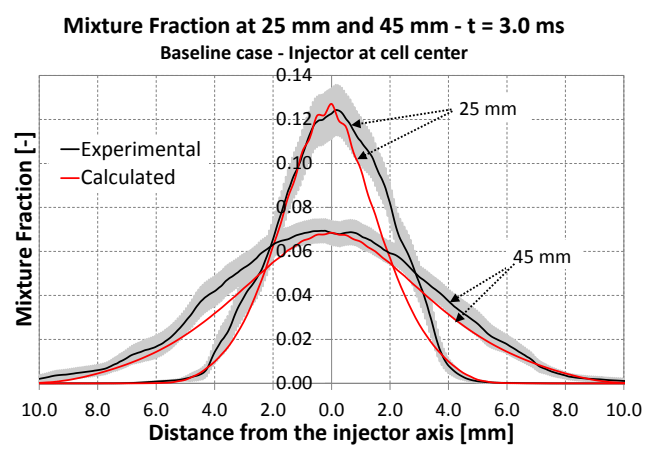

FIG. 4: Mixture Fraction at $25 \mathrm{~mm}$ and $45 \mathrm{~mm}$. Baseline
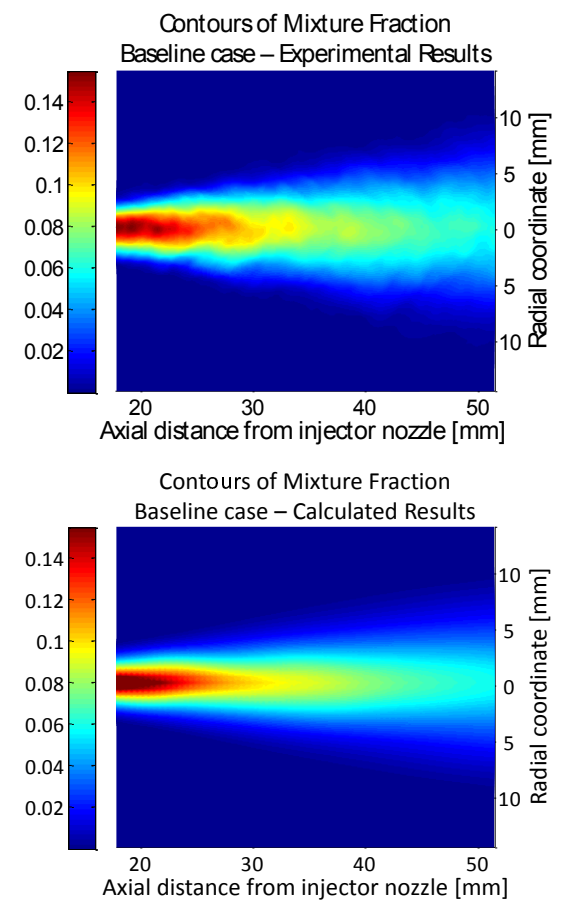

FIG. 5: Mixture Fraction contours. Baseline 


\subsection{SVI-DDM vs new model comparison}

Once defined the best setup, the baseline case was simulated on varying the injector position and comparing results of the SVI-DDM to those obtained with the standard DDM approach. In order to guarantee consistency, the same set of sub-models constant was used. As shown in Figs. 6 to 10 the standard approach is strongly affected by the choice of the injector location. This is due to the path followed by the parcels across the cells involved in the exchange processes. The case with standard DDM and nozzle at the vertex gives the most similar results to those obtained with the SVI-DDM: this happens because, injecting from a vertex and due to the disk injection (see Sec. 3.1), the parcels are uniformly distributed along four rows of cells so that the exchange of mass, momentum and energy is distributed on more cells than in the other two cases. In the same way the case with the nozzle in the center shows a larger penetration in terms of both liquid and vapor. The adoption of the new approach allows to obtain a very good independence on the nozzle position. The calculated liquid length is practically the same for all the cases and a more contained variability in jet penetration is observed. The new model allows also to reduce indirectly the fluctuations observed in the liquid penetration (Fig. 6). They are probably due to the reduced drag acting on the parcels when most of them interact with a smaller number of cells . Because of the locally higher momentum exchanged with the Eulerian domain, gas velocity increases and drag decreases. A lower drag slows less the parcels which penetrate more and create a spray where the liquid is more spread in the axial direction than in the radial. This, combined with a $99 \%$ threshold on liquid mass results in a more fluctuating liquid length.

Standard approach seems to catch better initial vapor penetration in corner and center cases. This is due to higher gas velocities that allow the jet to penetrate more in the domain. Even if at the beginning the jet penetration is better described, the higher velocities lead to overestimate the jet length in the steady state phase of injection. An increasing of mesh resolution in the near nozzle region (keeping an acceptable void fraction value) could represent better vapor penetration in the initial part of injection using the SVI-DDM. This should be able to catch velocity peaks that are probably smoothed by the adoption of a $0.5 \mathrm{~mm}$ minimum mesh size.

Figs. 8 to 9 show the axial profile of mixture fraction and axial component of gas velocity. It is clear that the mixture fraction is certainly described better by the SVI-DDM. Indeed, using the standard DDM and keeping the same constants setup could result (cell center case) in an inconsistent mixture fraction profile in the near-nozzle region. This behavior could be explained as follows: the first peak is mainly related to a strong creation of new droplets in the near nozzle region according to the $\mathrm{KH}$ breakup, the second peak is due to a delayed catastrophic breakup (RT mechanism) that takes place too far from the nozzle creating a non-physical distribution of mixture fraction and making the liquid penetrate more. The same behavior is shown also in the corner case, but with more limited consequences: since the liquid penetrates less than in the center case, but more than in the vertex one, mixture fraction shows a flat profile between 3 and 7 $\mathrm{mm}$ from the nozzle due to a slightly delayed catastrophic breakup.

Also the axial velocity profiles are less dependent on the injection position if the SVI-DDM is used. Having a reduced variability of axial velocity and smaller velocity gradients in the near-nozzle results in a good agreement of the three cases in the far-nozzle region, especially starting from $22-23 \mathrm{~mm}$ from the injector nozzle. The only noticeable difference in favor of the standard approach is that the vertex case predicts slightly better the mixture fraction distribution in the region at $18-20 \mathrm{~mm}$ from the nozzle. This happens because of the spherical control volumes around the parcel created by the SVI-DDM: in the vertex case the spheres ideally overlap around the injection axis concentrating the momentum exchange mainly in the cell of that area. This increases the gas velocity in that region, explaining the small differences at $18-20 \mathrm{~mm}$ in the axial profiles of the two vertex cases.

Fig. 9 shows that the SVI-DDM is able to reduce peak velocities in both the critical cases of standard approach. The reduction is more evident in the center case (about $20 \%$ ) while in the corner case is more contained (in the order of the 10\%). Because of lack of experimental data in the near nozzle region, nothing can be said about the predicted value, but the fact that, using the new approach, the variability of results is smaller can lead to more focused analysis of near nozzle region in future works. 

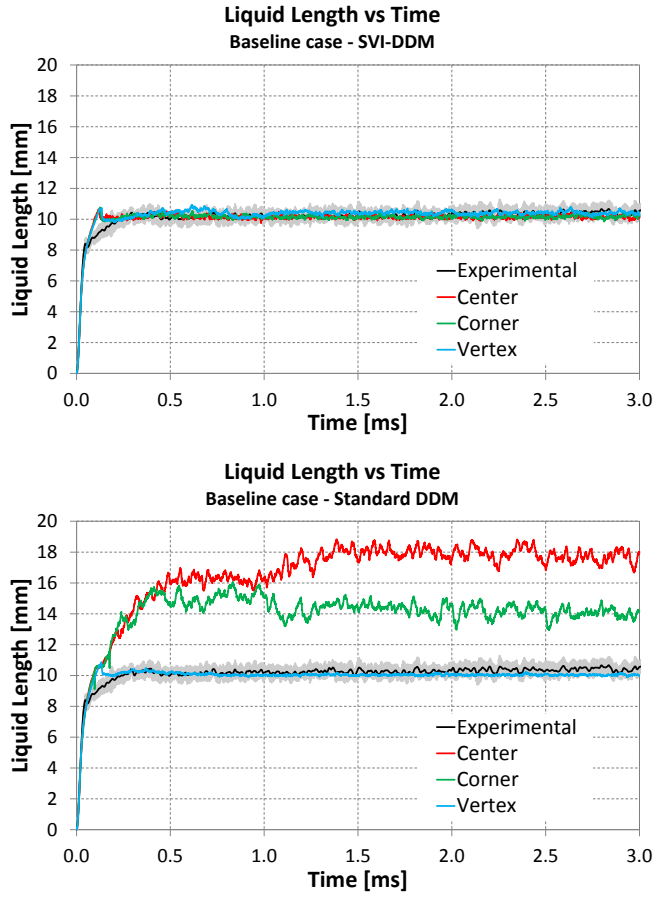

FIG. 6: Liquid Length on varying injector position
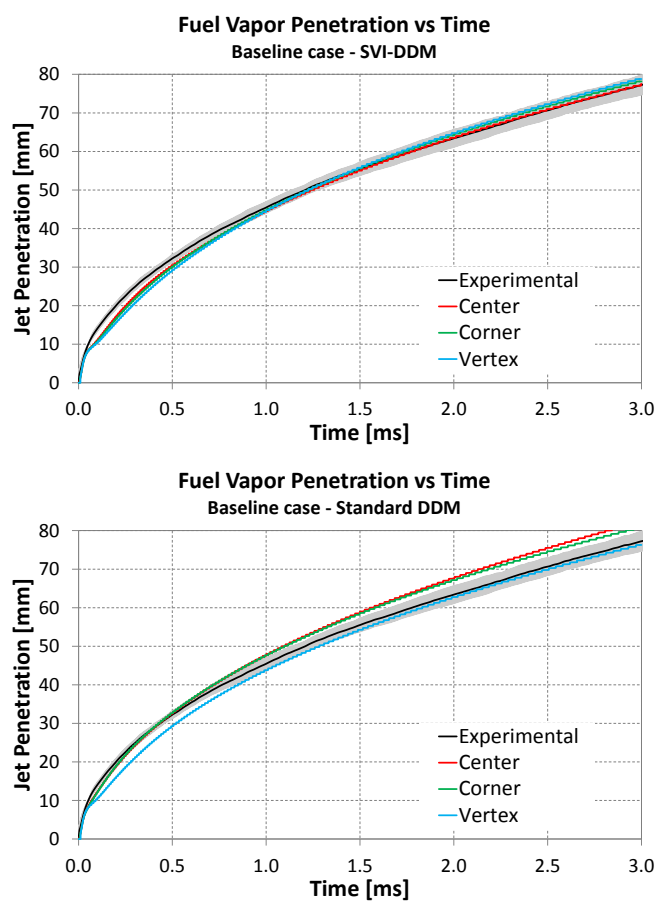

FIG. 7: Vapor Penetration on varying injector position
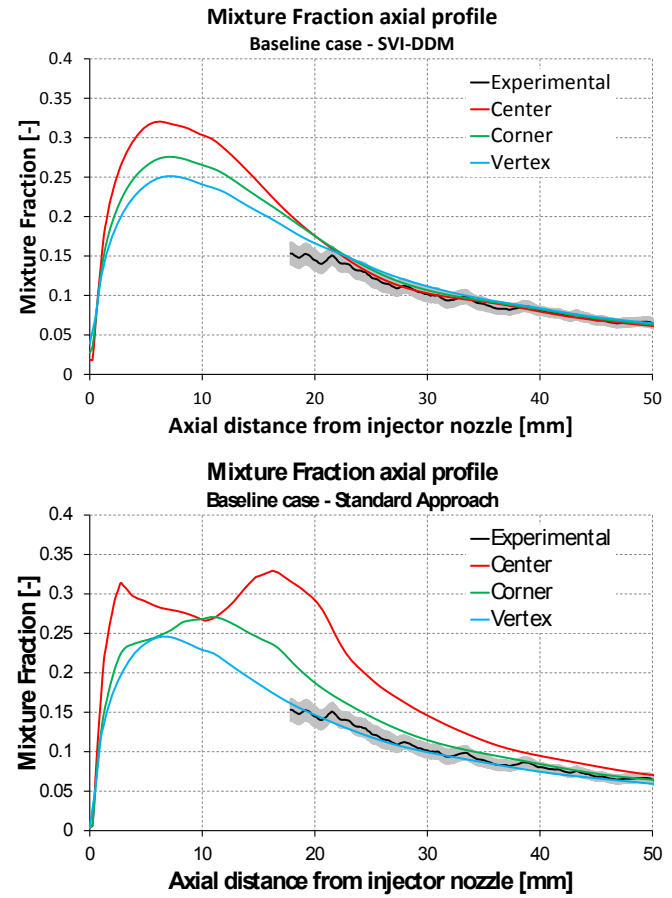

FIG. 8: Axial mixture fraction on varying inj. position
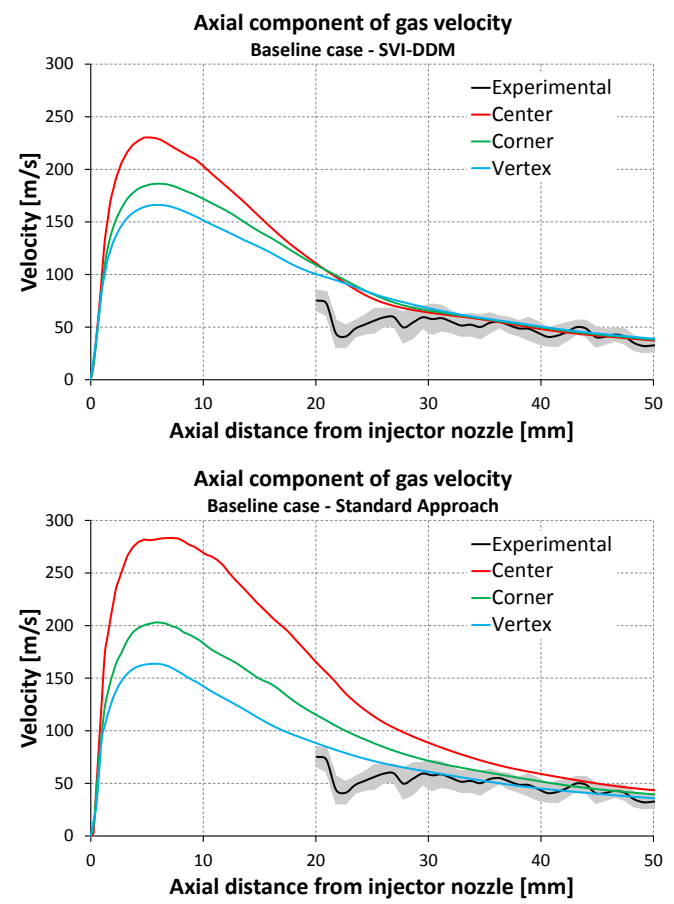

FIG. 9: Axial velocity profiles on varying inj. position 


\subsubsection{Global and local analysis of spray morphology for baseline conditions}

This section shows how the adoption of the SVI-DDM approach is effectively valid to reduce also the variability of global and local characteristics of the liquid spray morphology. Fig. 10 shows the global Sauter Mean Diameter vs time. As previously pointed out, corner and center positions with standard approach lead to have parcels interacting with less cells. This affects the drag that causes a lower deceleration of the parcels due to lower relative velocities. The correlation of the resulting wavelength in the RT breakup (APPENDIX A) shows that it increases as the deceleration of the parcel decreases making less probable the RT breakup mechanism itself and enhancing the $\mathrm{KH}$ breakup. At the same time KH usually creates very small parcels when liquid mass is shredded from the parent parcels resulting in a lower SMD.
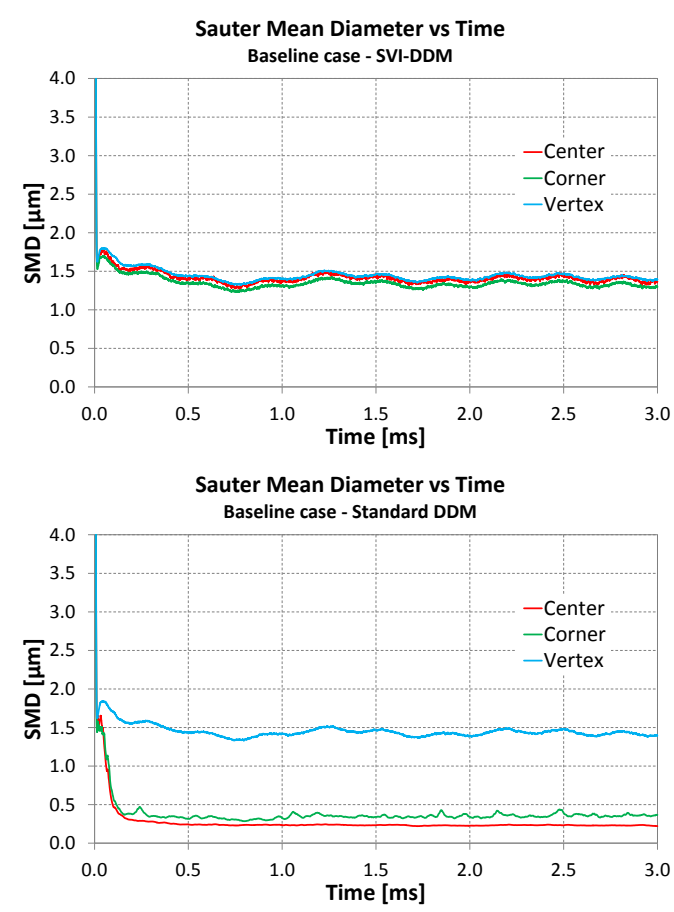

FIG. 10: SMD on varying injector position

The trend seen in Fig. 10 is confirmed by the analysis of the local droplet size distribution. Figs. 11 to 22 have been created at $1.0 \mathrm{~ms}$ after the start of injection (conditions of steady state liquid penetration). The results refers to all the droplets contained in a plane normal to the injection axis with a thickness equal to the minimum cell size and located at a user-defined distance from the nozzle.
The black bars represent the probability density function (PDF) of the droplet radius (scale is on the left), while the grey area shows the cumulative distribution function (CDF) calculated according to the liquid mass included in the considered slice (scale is on the right). As it is possible to see, a very contained variability of the shape of the $\mathrm{CDF}$ is observed with the new approach, furthermore the only case that shows similarities between the two DDM approaches is again the vertex case. The shapes of the CDFs help to explain also why the value of SMD is higher when SVI-DDM is used: only $10 \%$ of the liquid mass is included in parcels characterized by a very small radius. This value increases to $20-25 \%$ at $2.5 \mathrm{~mm}$ and to $55-60 \%$ at $5.0 \mathrm{~mm}$ when standard DDM is adopted consequently leading to a lower value of the global SMD.

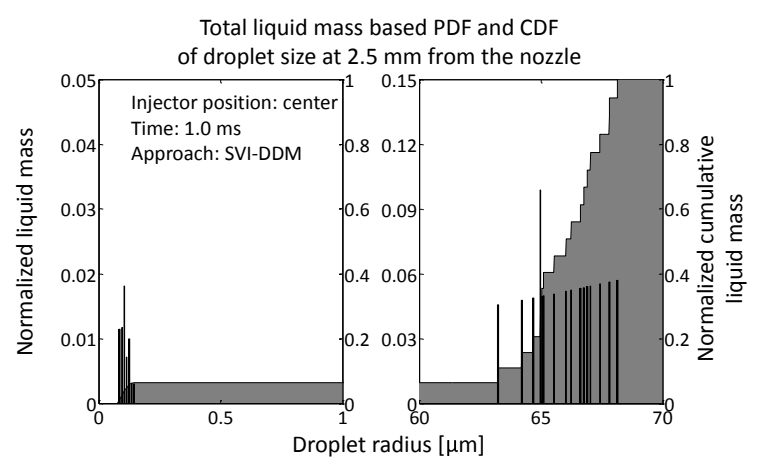

FIG. 11: Droplet size distribution at $2.5 \mathrm{~mm}$ from the injector nozzle. Center case with SVI-DDM

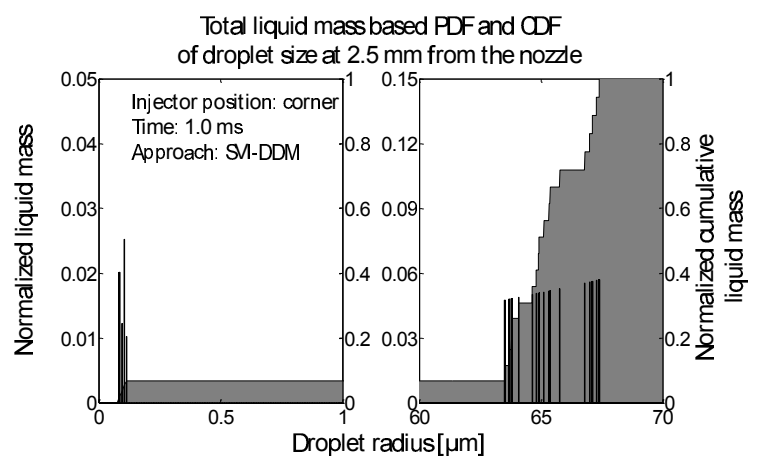

FIG. 12: Droplet size distribution at $2.5 \mathrm{~mm}$ from the injector nozzle. Corner case with SVI-DDM 


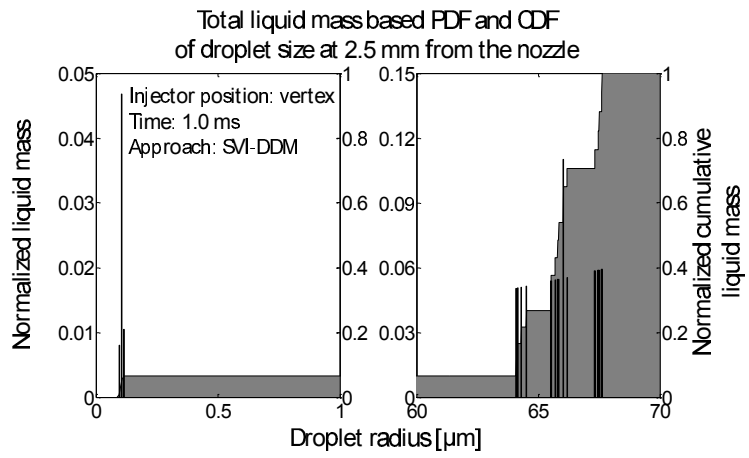

FIG. 13: Droplet size distribution at $2.5 \mathrm{~mm}$ from the injector nozzle. Vertex case with SVI-DDM

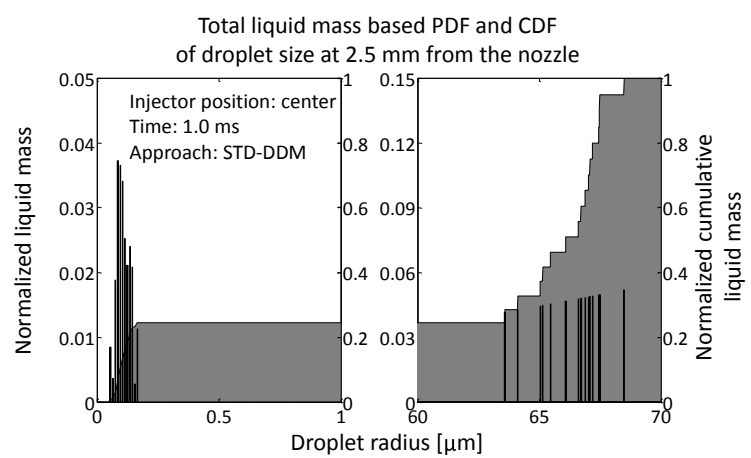

FIG. 14: Droplet size distribution at $2.5 \mathrm{~mm}$ from the injector nozzle. Center case with standard DDM

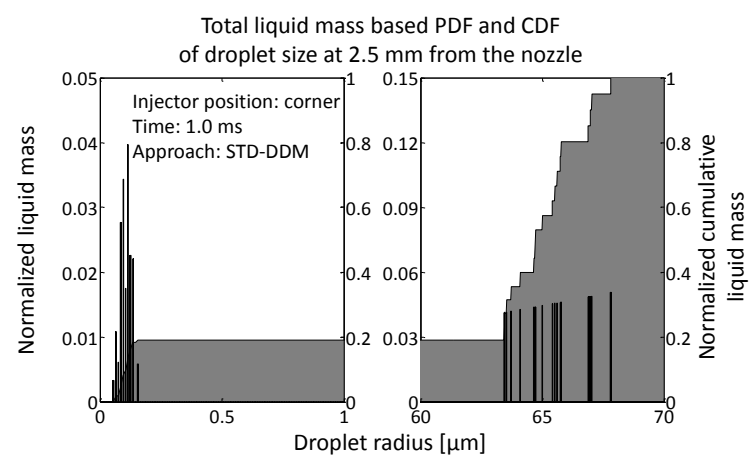

FIG. 15: Droplet size distribution at $2.5 \mathrm{~mm}$ from the injector nozzle. Corner case with standard DDM

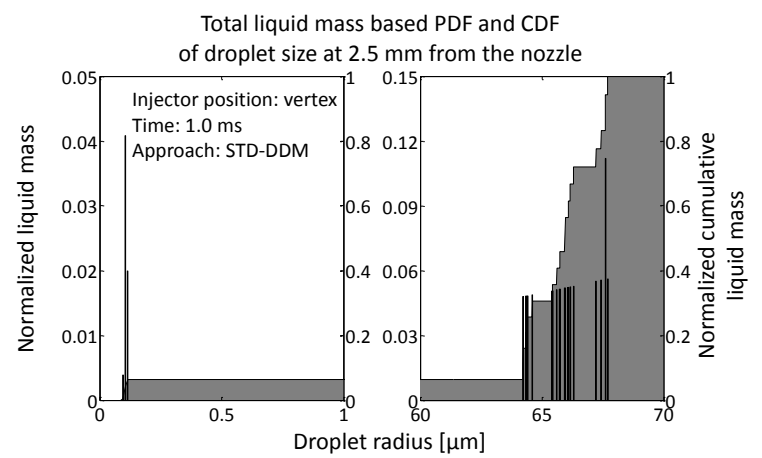

FIG. 16: Droplet size distribution at $2.5 \mathrm{~mm}$ from the injector nozzle. Vertex case with standard DDM

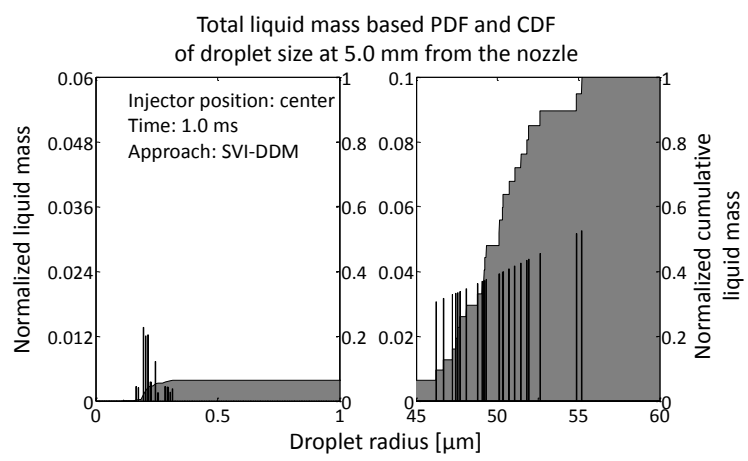

FIG. 17: Droplet size distribution at $5.0 \mathrm{~mm}$ from the injector nozzle. Center case with SVI-DDM

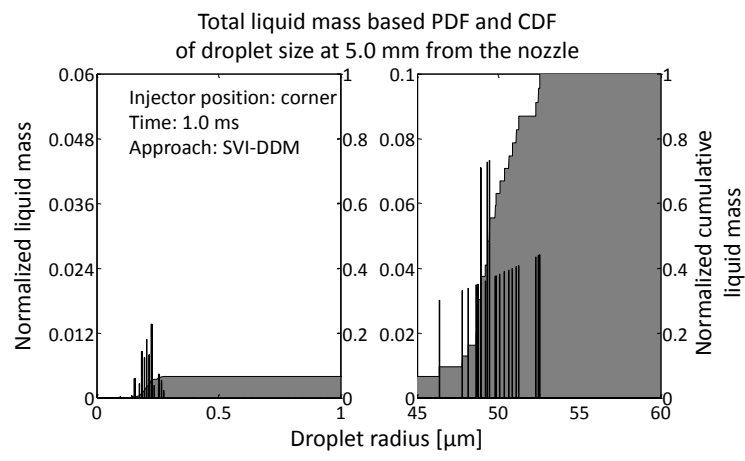

FIG. 18: Droplet size distribution at $5.0 \mathrm{~mm}$ from the injector nozzle. Corner case with SVI-DDM 


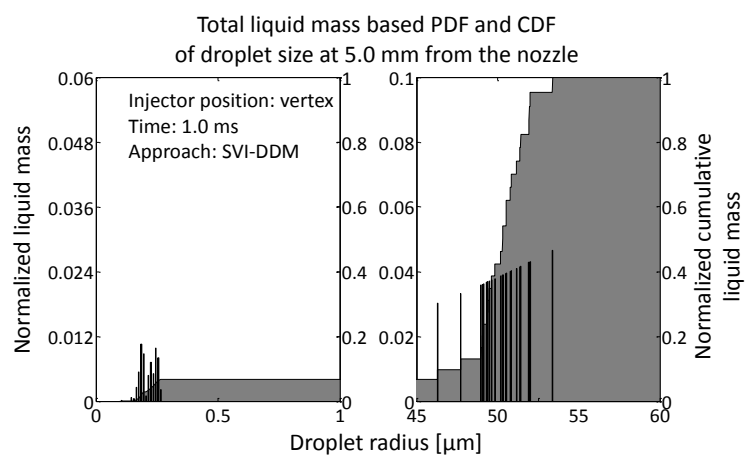

FIG. 19: Droplet size distribution at $5.0 \mathrm{~mm}$ from the injector nozzle. Vertex case with SVI-DDM

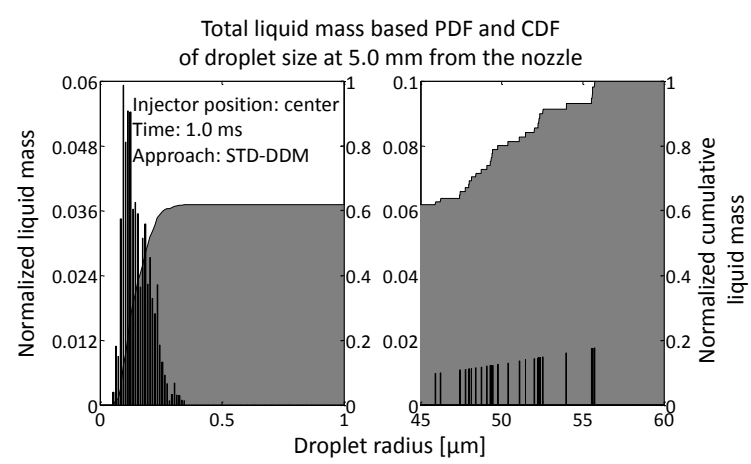

FIG. 20: Droplet size distribution at $5.0 \mathrm{~mm}$ from the injector nozzle. Center case with standard DDM

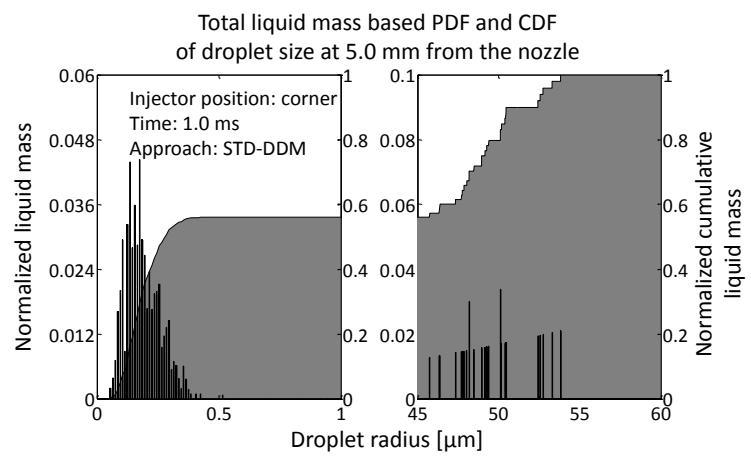

FIG. 21: Droplet size distribution at $5.0 \mathrm{~mm}$ from the injector nozzle. Corner case with standard DDM

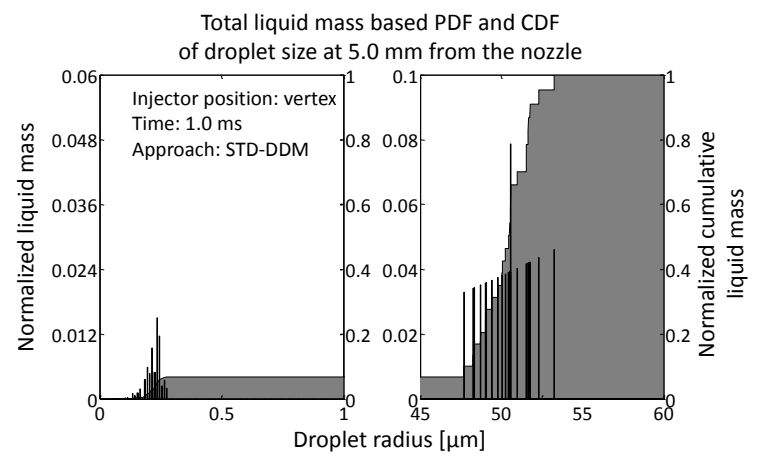

FIG. 22: Droplet size distribution at $5.0 \mathrm{~mm}$ from the injector nozzle. Vertex case with standard DDM

\subsection{Model response to ambient density variation}

The same analysis of sec. 5.3 was performed using different ambient conditions. The density was decreased from 22.8 to 15.2 and $7.6 \mathrm{~kg} / \mathrm{m}^{3}$, keeping constant ambient temperature. The tunable parameters were left the same as they were defined after matching experimental baseline results. The only available experimental measurement for the two cases was the vapor penetration and, as showed in Figs.23 to 25, both approaches were able to respond to the changed ambient conditions. The only inconsistency was identified in a flat trend of liquid length with standard DDM when the ambient density was increased from 15.2 to $22.8 \mathrm{~kg} / \mathrm{m}^{3}$. This could be seen as a confirmation that using the standard DDM approach is necessary to tune the models every time the injector position changes in order to avoid a wrong estimation of the momentum exchange. As for the baseline case, the SVI-DDM showed a more contained variability of the results with respect to the injector position for what concerns vapor penetration. Also computed liquid length (see Fig.23) showed a similar behavior as for the baseline case, indeed is shown that this quantity was practically independent on the injector position responding only to the ambient density variation. Furthermore, even if experimental measurements of liquid length were not available for the simulated injector, the found average value was in accordance to the trend shown in the work of García-Oliver et al. (2013). In that work the authors simulated a similar injector in comparable conditions to those of the present work. 


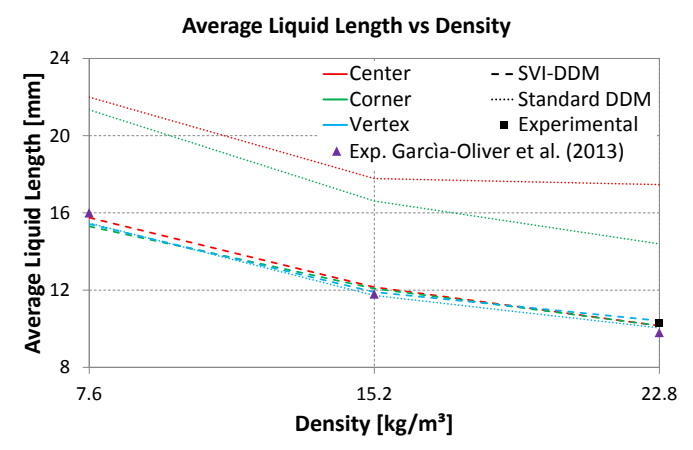

FIG. 23: Average Liquid Length on varying injector position and ambient density

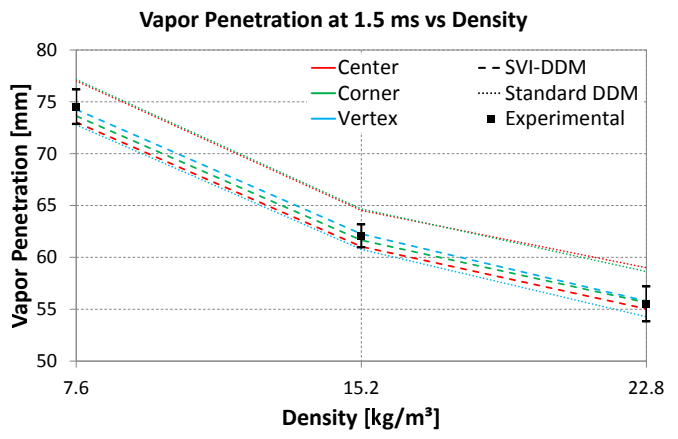

FIG. 24: Vapor Penetration at $1.5 \mathrm{~ms}$ on varying injector position and ambient density

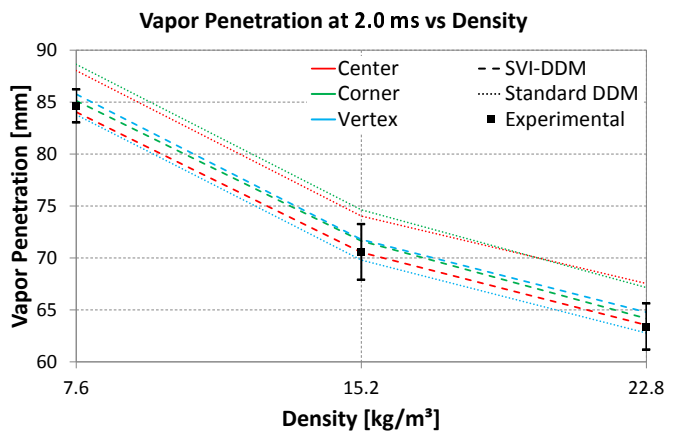

FIG. 25: Vapor Penetration at $2.0 \mathrm{~ms}$ on varying injector position and ambient density

\subsection{Model response to injection pressure varia- tion}

The analysis made in this section aims to show how the two approaches respond to a change of the injection pressure. As done for most of the previous analysis, the experimental data were taken from the SANDIA ECN database (Sandia ECN website, 2013), while the three injection laws used to simulate the injection pressure variation were generated by means of an algorithm made available by CMT (CMT website, 2013). This algorithm is able to generate a coherent injection law, starting from injection and ambient pressures, nozzle diameter, nozzle discharge coefficient, fuel type and injection duration. As done for the previous sections the results of Figs. 26 to 28 are shown on varying the injector position within the hosting cell.

Fig.26 confirms again that the SVI-DDM approach is able to guarantee a very contained variability in terms of calculated average liquid length. As for the previous cases the most similar results between the two models can be obtained only if the injector is placed in a cell vertex when the standard DDM is used. Both models predict a correct liquid length for the $100 \mathrm{MPa}$ case, while in the $50 \mathrm{MPa}$ case a slightly larger value results. Figs. 27 and 28 show a more contained variability of the calculated jet length when the new DDM approach is used. The vapor penetration is a little under-estimated with respect to experimental results, but the general trend is well caught. The differences in liquid and vapor penetration could be due to choice to use an algorithm for generating the injection law that does not take in account that the real law used in the experiments could be slightly different from the one here adopted. As seen for the density variation analysis, the case with injector in the center and standard DDM is the most critical. The liquid length shows a divergent trend going from 100 to $150 \mathrm{MPa}$. Again, the probable explanation is that the use of a wrong set of models constants leads to a wrong computation of the momentum exchange between the two phases. 


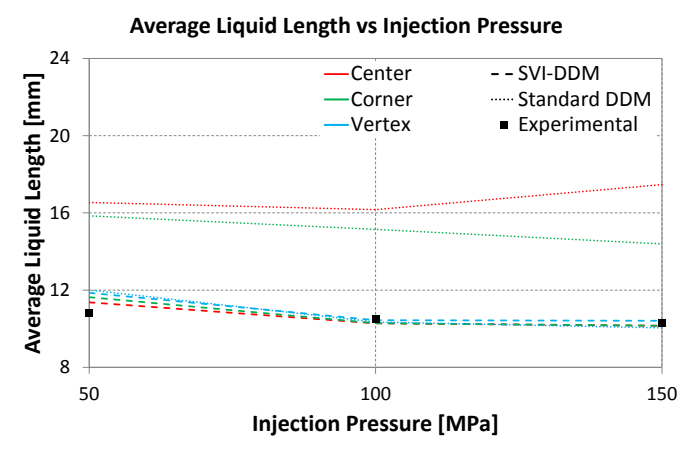

FIG. 26: Average Liquid Length on varying injector position and injection pressure

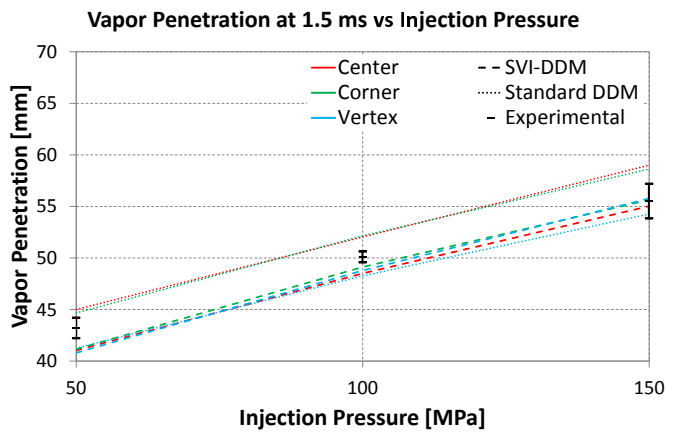

FIG. 27: Vapor Penetration at $1.5 \mathrm{~ms}$ on varying injector position and injection pressure

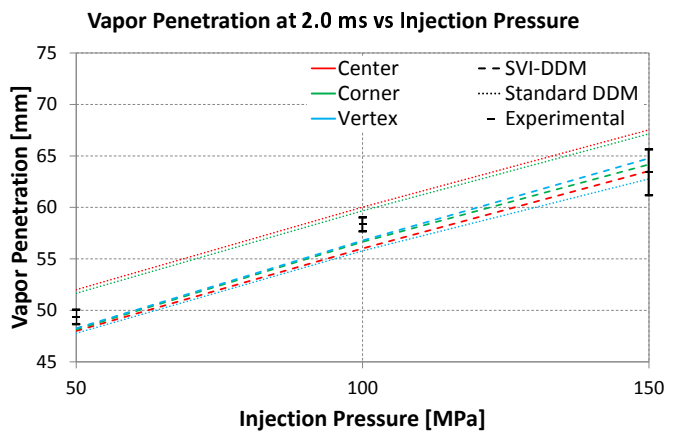

FIG. 28: Vapor Penetration at $2.0 \mathrm{~ms}$ on varying injector position and injection pressure

\subsection{Spray-grid mutual orientation analysis}

In order to test and compare the sensitivity of the two approaches to the spray-grid mutual orientation, a new mesh was created in which the mesh elements were rotated by $45^{\circ}$ with respect to the injector axis. This was done to make the parcels interacting with a mesh not-aligned with the spray. Having a mesh not aligned with the spray means that there is not a preferential lane in which the spray can evolve. This should reduce the dependency on the injector nozzle also using standard DDM approach. The aim of the analysis described in this section is to test how the two approaches react to the changed mesh orientation. In this case, changing the position from the cell center to the vertex does not make a significant variation of the cells interacting with the spray, so two new injectors positions replaced the "vertex" and "corner" cases. On this meshes, the injector was placed in the cell center as reference case and then it was moved to a face center and to an edge center(Fig. 29).

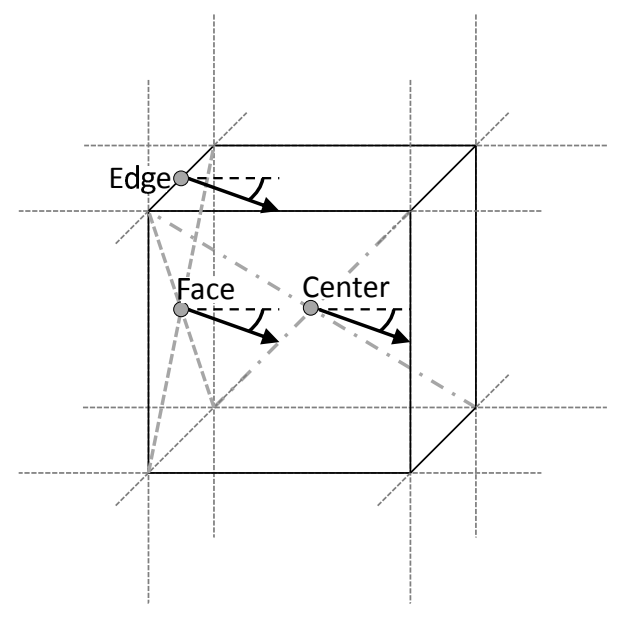

FIG. 29: Injection positions and directions. $45^{\circ}$ cases

The most critical case among the three is the "edge center" because the spray involves two planes of cells, while in the other two cases it interacts mainly with the plane containing the hosting cell and the injection axis. Figs. 30 to 31 show that using the same setup as for the baseline case, an under-estimated vapor penetration is observed for both the approaches, while liquid length is not strongly affected by the mesh orientation. The reason of this behavior could be related to the presence of a mesh-induced false diffusion (Vahl and Mallinson, 1972). Due to the orientation of the mesh cells with respect to the spray, the fluxes of the gaseous phase present a com- 
ponent that is normal to the injection axis and tends to enlarge the jet to the sides and to shrink it in the axial direction (see Fig.32). This difference could be limited by tuning the turbulence model in order to have a reduction in the turbulent viscosity that could allow a larger fuel jet penetration or by adopting a higher order solution scheme possibly coupled with a smaller time step. Nevertheless the adoption of the SVI-DDM model resulted in a more contained variability especially in the near-nozzle region during the first phase of the injection. Indeed it is clear how the three curves are practically overlapped in the first millisecond of the injection, where the jet tip reaches a maximum penetration of $40 \mathrm{~mm}$. As opposite the standard DDM approach showed divergent fuel jet penetrations since the very first part of the injection (see Fig. 31). Also the trend showed among the three cases is consistent with the simulations with the spray-aligned mesh. Here the edge case is the more similar to the vertex case because of the reasons explained before and as before it is the one that shows the largest penetration with SVI-DDM and the lowest with standard approach.
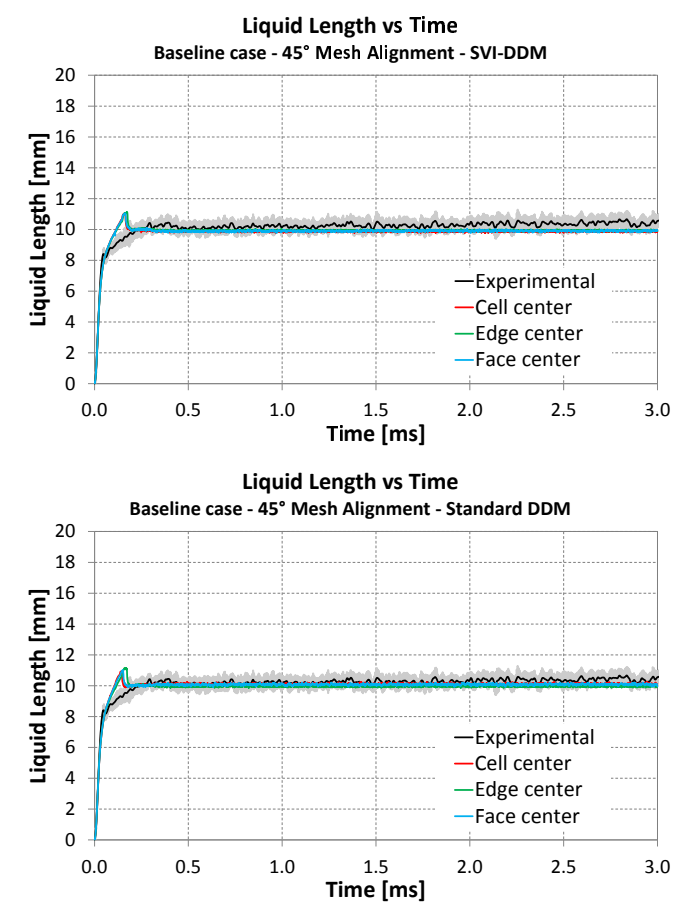

FIG. 30: Liquid Length on varying injector position
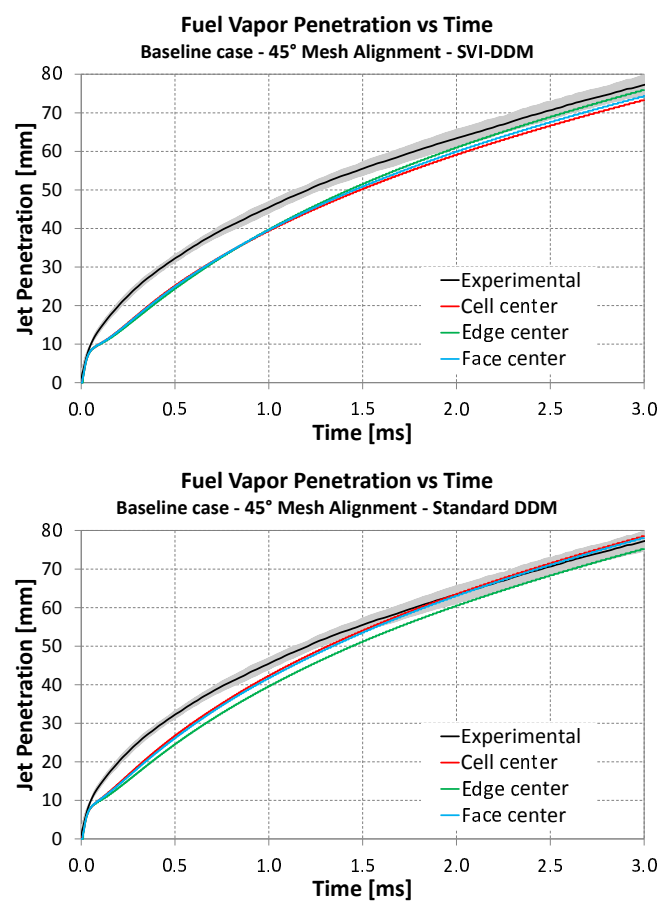

FIG. 31: Vapor Penetration on varying injector position

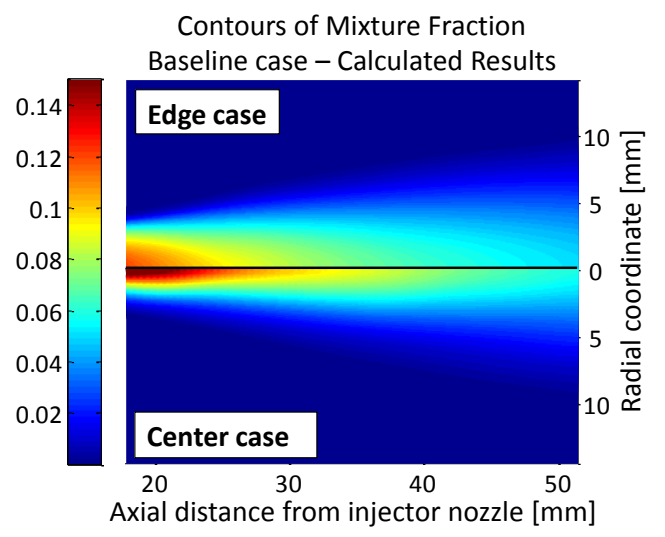

FIG. 32: Mixture Fraction contours. Comparison between Baseline Center and Edge cases using SVI-DDM approach 


\subsection{Application on an engine geometry}

The last part of this work has been dedicated to test the SVI-DDM approach on a simplified engine geometry in non-reacting conditions. The mesh was created starting from the geometry of a Cat Engine (C7.1 model). For the purposes of this study an axi-symmetrical domain was preferred hence the head details were simplified in a flat surface and a crevices volume was introduced to preserve the compression ratio. All the meshes used for this simulation were created by means of the OpenFOAM utility snappyHexMesh (OpenFOAM website, 2013). Starting from the beginning of the simulation every mesh has its own range of validity expressed as the crank angle interval along which the mesh is moved and deformed. Once the limit of validity of the mesh is reached, all the information contained in the Eulerian domain are mapped on the new following mesh. SnappyHexMesh is a tool that allows to create cartesian meshes automatically by means of a pre-meshed prismatic block and a surface file containing the information of the geometry. This means that the main structure of the mesh will be defined by how the block is discretized. The CFD domain will be the result of the intersection of the surface file with the block and the elements that are located on this intersection will be snapped to catch as well as possible the shape of the surface. Furthermore it is also possible to locally refine the mesh with respect to user-defined regions and surfaces. In this case the mesh was refined in the region in which the liquid spray was thought to interact with the gaseous phase in order to have a minimum mesh size of $0.5 \mathrm{~mm}$. As already pointed out in sec. 4.1 , the choice to use this value is mainly related to the necessity to keep the number of cells in a range between two and three millions elements. Due to the size of this engine, a further refinement would have resulted in an increase in the amount of cells that was considered not acceptable for the aim of this the study. Fig. 33 shows half of the geometry at $10^{\circ}$ before the TDC. It is possible to see the refinement region around the injector, furthermore the Lagrangian phase of one of the four simulated sprays is shown to give an idea of its trajectory across the domain.

\subsubsection{Description of the simulation and results}

The simulation started at the Intake Valve Closing using an experimental set of given thermo-physical initial conditions. In order to keep the axial symmetry, the flow field has been initialized neglecting the presence of any swirl motion. This means that the velocity field at the start of

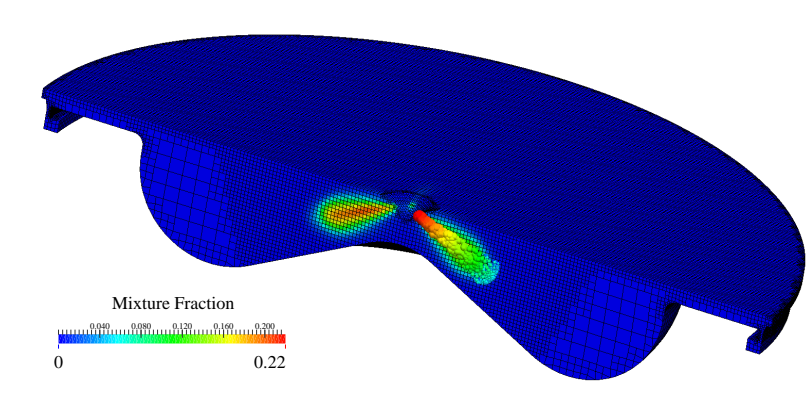

FIG. 33: Engine simulation. Detail of the grid

injection was only related to the piston motion during the compression phase. The Table 3 summarizes the main data of the engine simulation. These data were provided by Caterpillar UK Engines Company Ltd. Four nozzles were arranged along the injector tip forming an angle of $90^{\circ}$ between each nozzle and the next. As done in part of the previous analysis on the Sandia constant volume vessel, the attention was focused on mixture fraction and gas velocity. The graphics reported in Figs.34 to 35 show some comparisons of the profile of evaporated fuel and axial velocity component along the centerline of every spray axis for each individual nozzle. The profiles are extracted at 3 crank angle degrees before the TDC. Due to the shape of the spray cone angle the trajectories of the liquid fuel across the domain did not follow preferential paths within the mesh. This is an advantageous condition for the standard approach for the reasons explained in Sec. 2 and confirmed in Sec.5.6. Nevertheless Figs. 34 and 35 show how the SVI-DDM approach is able to guarantee more similar profiles in terms of mixture fraction distribution and velocity. This can be seen as a confirmation that the new model is less grid-dependent in terms of exchange of momentum and mass from the liquid to the gaseous phase. Moreover the velocity generally results a little lower in the case of the new approach as it was pointed out with the spray A results. This is probably due to the different exchange of momentum that involves more than one cell at the same time according to how the grid is intersected by the sphere. The SVI-DDM tends to diffuse momentum to the sides and consequently reduces the peak value along the injection axis. Standard DDM shows also that the predicted mixture fraction and gas velocity in the near-nozzle region strongly differs from nozzle to nozzle. This difference are almost completely canceled when the SVI-DDM is adopted. 

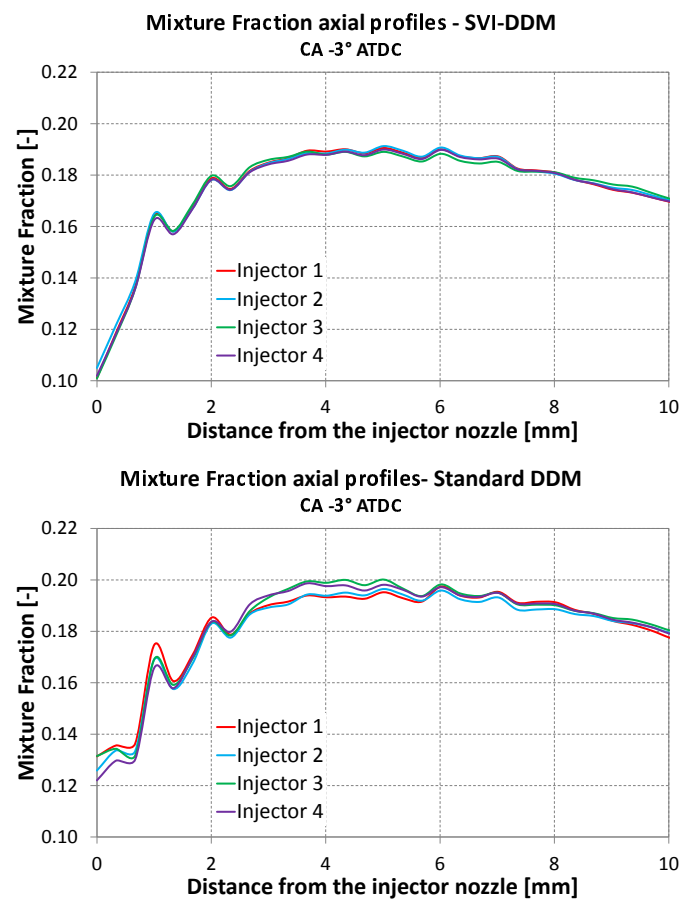

FIG. 34: Mixture Fraction axial profile at $-3^{\circ}$ aTDC.
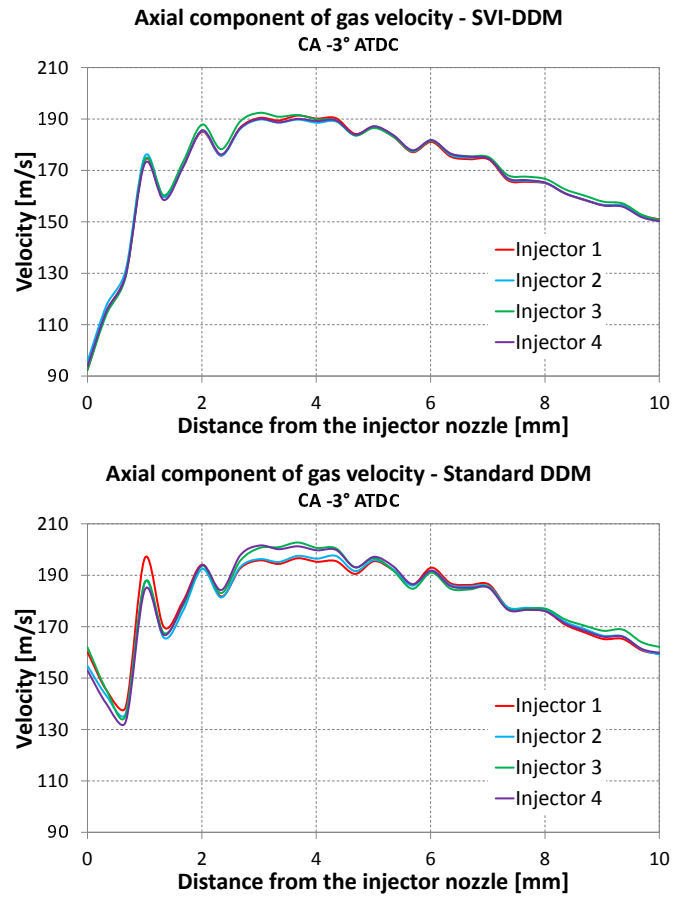

FIG. 35: Gas velocity along the injection axis at $-3^{\circ}$ aTDC.
TABLE 3: Engine and injection data

\begin{tabular}{|c|c|c|c|}
\hline \multicolumn{2}{|c|}{ Engine Specification } & \multicolumn{2}{c|}{ Fuel Injection } \\
\hline Bore $[\mathrm{mm}]$ & 105 & Start Of Injection - SOI [ ${ }^{\circ} \mathrm{CA}$ aTDC] & -14 \\
\hline Stroke $[\mathrm{mm}]$ & 127 & Injection Duration $\left[{ }^{\circ} \mathrm{CA}\right]$ & 29.65 \\
\hline Engine Speed $[\mathrm{rpm}]$ & $\mathbf{2 2 0 0}$ & No. Of Nozzle Holes & 4 \\
\hline IVC $\left[{ }^{\circ} \mathrm{CA}\right.$ aTDC] & -146 & Nozzle Hole Diameter [mm] & 0.152 \\
\hline
\end{tabular}

\section{CONCLUSIONS}

A novel approach based on a spherical volume interaction between the liquid parcels and the gaseous phase was proposed and compared to the standard DDM approach for Diesel spray modeling. The main scope of the proposed contribution was to reduce the dependency of the momentum exchange between the Lagrangian and Eulerian fields on the injector location and direction with respect to the grid. Comparison with the standard approach were carried on considering both constant volume vessel experiment and a Diesel engine. Results showed a significant reduction of dependency of the liquid length penetration on the injector position in the grid cells and less evident but still appreciable improvements on the reduction of the dependency of the vapor phase morphology. For what concerns the spray/grid orientation effects, a deep investigation is required to evaluate and propose possible solutions that could allow reducing the dependency when the parcels cross the domain following a diagonal path with respect to cells alignment. The new SVI-DDM approach showed that it is able to reduce nozzle-to-nozzle dependency within the same calculation even in those cases, given that all the liquid jets are oriented in a similar fashion. Bigger differences could be surely found if different orientations should be adopted within the same calculation.

\section{ACKNOWLEDGEMENTS}

The authors would like to acknowledge Caterpillar Inc. for providing the grant that made this work possible. 


\section{APPENDIX A. KHRT BREAK-UP MODEL}

The implementation of the KHRT model adopted in this work combines two models in a competing manner: the Kelvin-Helmoltz (KH) and the Rayleigh-Taylor (RT). The first model applied by the algorithm is the RT. If the conditions to apply it are not satisfied, the algorithm proceeds to apply the $\mathrm{KH}$ routine.

In the near nozzle region the governing breakup mechanism is the one modeled by the Kelvin-Helmoltz theory. Reitz (1987) showed that the wave-breakup theory describing the development of $\mathrm{KH}$ instabilities on a jet surface can be applied to model both primary and secondary atomization of droplets. In KH model the breakup time is computed as follows:

$$
\tau_{\mathrm{KH}}=3.788 B_{1} \frac{r_{p}}{\Lambda_{\mathrm{KH}} \Omega_{\mathrm{KH}}}
$$

where $\Lambda_{\mathrm{KH}}$ and $\Omega_{\mathrm{KH}}$ are respectively wavelength and maximum growth rate of the liquid surface perturbations usually caused by effects of the inner nozzle flow, e.g. by turbulence within the liquid phase. The full analytical formulation can be found in Reitz (1987) who proposed also the curve-fits of numerical solutions that are reported here:

$$
\begin{gathered}
\Lambda_{\mathrm{KH}}=9.02 r_{p} \frac{(1+0.45 \sqrt{\mathrm{Oh}})\left(1+0.4 \mathrm{Ta}^{0.7}\right)}{\left(1+0.865 \mathrm{We}_{g}^{1.67}\right)^{0.6}} \\
\Omega_{\mathrm{KH}}=\frac{\left(0.34+0.38 \mathrm{We}_{g}^{1.5}\right)}{(1+\mathrm{Oh})\left(1+1.4 \mathrm{Ta}^{0.6}\right)} \sqrt{\frac{\sigma}{\rho_{l} r_{p}^{3}}}
\end{gathered}
$$

During the $\mathrm{KH}$ routine the parent parcel can undergo two different mechanisms. The first is the enlargement of the droplet that can only occur if simultaneously $\tau_{\mathrm{KH}}$ is larger than the perturbations lifetime and the wavelength satisfies the relation $r_{p}<B_{0} \lambda_{\mathrm{KH}}$ (whit $B_{0}$ constant of the $\mathrm{KH}$ model usually equal to 0.61 ). This mechanism can occur only once for each parcel and only if the other mechanisms have not occurred yet.

$$
r_{p, \text { new }}=\min \left\{\begin{array}{l}
\sqrt[3]{3 \pi r_{p}^{2} \frac{\mathbf{U}_{\text {rel }}}{2 \Omega_{\mathrm{KH}}}} \\
\sqrt[3]{3 r_{p}^{2} \frac{\Lambda_{\mathrm{KH}}}{4}}
\end{array}\right.
$$

The second mechanism in $\mathrm{KH}$ is also the main one. If the child droplets radius $r_{c}=B_{0} \lambda_{\mathrm{KH}}$ is smaller than the parent droplet radius the $\mathrm{KH}$ algorithm checks if stripping breakup can occur. Due to the breakup and generation of new small droplets, the size of the original droplet is reduced. The temporal change in diameter of the parent droplet is given by:

$$
\frac{d r_{p}}{d t}=-\frac{r_{p, \text { new }}-r_{c}}{\tau_{\mathrm{KH}}}
$$

from which through an implicit integration it is possible to obtain the updated radius of the parent droplet:

$$
r_{p, \text { new }}=\frac{f r_{c}+r_{p}}{1+f}
$$

with $f=\Delta t / \tau_{\mathrm{KH}}$.

The stripped mass is calculated as:

$$
m_{s}=m_{p}\left(1-\frac{d_{p, n e w}^{3}}{d_{p}^{3}}\right)
$$

whit which it is possible to calculate the number of droplets in the child parcel:

$$
N_{c}=\frac{6 m_{s}}{\rho_{l} \pi d_{c}^{3}}
$$

If the number of child droplets, $N_{c}$, is larger than the number of droplets contained in the parent parcel, $N_{p}$, the breakup occurs and a new parcel of mass $m_{s}$ is generated.

To save computational resources the stripped mass is accumulated till it reaches the $3 \%$ of the parent parcel mass (Patterson and Reitz, 1998), when the breakup occurs. During and after this phase the total number of droplets in the parent parcel is conserved. This means that a parcel that undergoes only $\mathrm{KH}$ breakup will have always the same number of droplets assigned at the moment of the injection. This is done because the idea is to describe the stripping breakup as a detachment of mass from the parent droplet to create new droplets. Since in a parcel the included droplets are all equal and they undergo the same mechanisms, the behavior has to be the same and they have to generate at least one child droplet each.

Once a new parcel is generated it is given the same temperature and physical location as the parent and the radius is given $r_{c}=B_{0} \lambda_{\mathrm{KH}}$. It is given the same velocity in terms of magnitude but it is also introduced a small deviation from the original direction by means of casual generation of the two components in the plane orthogonal to the parent parcel direction.

Downstream of the nozzle Rayleigh-Taylor breakup takes place. The RT breakup model is based on the theoretical considerations of Taylor (1963) who investigated 
the stability of liquid-gas interfaces when accelerated in a normal direction with respect to the plane that contains them. If the directions of acceleration and density gradient are concordant the interface will result stable, otherwise Rayleigh-Taylor instabilities can develop. In the case of a liquid droplet decelerated by drag forces in a gas phase, these instabilities may grow at the trailing edge of the droplet.

The droplet acceleration is expressed as:

$$
\mathbf{a}_{d}=\frac{3}{8} C_{d} \frac{\rho_{g}}{\rho_{l}} \frac{\left|\mathbf{u}_{r e l}\right|^{2}}{r}
$$

where $C_{d}$ is the drag coefficient and $\mathbf{u}_{r e l}$ is the relative velocity between liquid and gas. The gas velocity is interpolated at the parcel position using the technique explained in sec. 2.7. By means of the droplet acceleration it is possible to calculate the frequency and wavelength of the fastest growing waves:

$$
\begin{array}{r}
\Omega_{\mathrm{RT}}=\sqrt{\frac{2}{3 \sqrt{3 \sigma}} \frac{\left[\left|\mathbf{a}_{d}\right|\left(\rho_{l}-\rho_{g}\right)\right]^{3 / 2}}{\rho_{l}+\rho_{g}}} \\
\Lambda_{\mathrm{RT}}=C_{\mathrm{RT}} 2 \pi \sqrt{\frac{3 \sigma}{\left|\mathbf{a}_{d}\right|\left(\rho_{l}-\rho_{g}\right)}}
\end{array}
$$

In the eqs. A.10 and A.11 $\sigma$ is the surface tension and $C_{\mathrm{RT}}$ is a tunable constant to allow a modification of the effective wavelength. It accounts for unknown effects of initial conditions like turbulence and cavitation inside the nozzle on the secondary breakup. RT breakup occurs only if the wavelength $\Lambda_{R T}$ is smaller than the the droplet diameter and if the lifetime of the instabilities is greater than a breakup time calculated as:

$$
\tau_{\mathrm{RT}}=\frac{C_{\tau}}{\Omega_{\mathrm{RT}}}
$$

with $C_{\tau}$ as a tunable constant for reducing or increasing the break-up time to match experimental results.

Furthermore in this implementation of the KHRT, the use of a breakup length was made. RT breakup is completely excluded if the distance between the parcel and the injector nozzle is smaller than a certain threshold calculated as follow:

$$
L_{b u}=C_{b u} d_{n o z} \sqrt{\frac{\rho_{l}\left(T_{i n j}\right)}{\rho_{g}}}
$$

Here $C_{b u}$ is a tunable constant and the liquid density value is taken at the injection temperature. The use of a breakup length is justified in the works of Chan et al. (1997), Powell et al. (2001) and Linne et al. (2006): experimental results have shown that diesel spray penetration has different rates within and beyond a breakup length near the nozzle exit and this may be attributed to the existence of an intact liquid core close to the nozzle. The used threshold allows to catch better this behavior.

The Table A. 4 summarizes the values adopted for each constants present in the KHRT breakup model:

TABLE A.4: KHRT breakup model constants

\begin{tabular}{|c|c|c|c|c|}
\hline$B_{0}$ & $B_{1}$ & $C_{\mathrm{RT}}$ & $C_{\tau}$ & $C_{b u}$ \\
\hline 0.61 & 25 & 0.2 & 0.2 & 25 \\
\hline
\end{tabular}

\section{REFERENCES}

Abani, N., Munnannur, A., and Reitz, R. D., ICEF2007-1667. Reduction in numerical parameter dependencies in diesel spray models, Proc. ASME Internal Combustion Engine Division, Charleston, South Carolina, USA, 2007.

Abraham, J. and Magi, V., A virtual liquid Source (VLS) model for vaporizing Diesel Sprays, SAE Technical Paper 1999-010911, 1999.

Baumgarten, C., Mixture formation in internal combustion engines, Springer, 2006.

Chan, M., Das, S., and Reitz, R., Modeling multiple injection and EGR effects on diesel engine emissions, SAE Technical Paper 972864, 1997.

CMT website, CMT-Motores Trmicos, Universitat Politenica de Valncia, November, 2013.

URL http: / / www . cmt . upv. es/ECN03. aspx

Crowe, C., Sommerfeld, M., and Tsuji, Y., Multiphase flows with droplets and particles, CRC Press LLC, 1998.

D'Errico, G., Ettorre, G., and Lucchini, T., Comparison of combustion and pollutant emission models for DI diesel engines, SAE Technical Paper 2007-24-0045, 2007.

D'Errico, G., Lucchini, T., Atzler, F., and Rotondi, R., Computational fluid dynamics simulation of diesel engines with sophisticated injection strategies for in-cyinder pollutant controls, Energy and fuels, vol. 26, pp. 4212-4223, 2012.

Dukowitz, J., A particle fluid numerical model for liquid sprays, Journal of Computational Physics, vol. 35, pp. 229-253, 1980.

Ferziger, H. J. and Perik, M., Computational methods for fluid dynamics, Springer, 2002.

García-Oliver, J. M., Pastor, J. M., Pandal, A., Trask, N., Baldwin, E., and Schmidt, D., Diesel spray CFD simulations based on the Sigma-Y eulerian atomization model, Atomization and Sprays, vol. 23, pp. 71-95, 2013. 
Huang, C. and Lipatnikov, A., Modeling of gasoline and ethanol hollow-cone sprays using OpenFOAM, SAE Technical Paper 2011-01-1896, 2011.

Issa, R. I., Solution of the implicitly discretized fluid flow equations by operator-splitting, Journal of Computational Physics, 1986.

Jasak, H., Error analysis and estimation for the finite volume method with applications to fluid flows, $\mathrm{PhD}$ thesis, Imperial College of Science, Technology and Medicine, 1996.

Kosaka, H., Suzuki, T., and Kamimoto, T., Numerical simulation of turbulent dispersion of fuel droplets in an unsteady spray via discrete vortex method, SAE Technical Paper 952433, 1995.

Kösters, A. and Karlsson, A., A comprehensive numerical study of diesel fuel spray formation and combustion in OpenFOAM, SAE Technical Paper 2011-01-0842, 2011.

Kralj, C., Numerical simulation of Diesel spray processes, $\mathrm{PhD}$ thesis, Imperial College of Science, Technology and Medicine, 1995.

Launder, B. E. and Sharma, B. I., Application of the energy dissipation model of turbulence to the calculation of flow near a spinning disc, Letters in heat and mass transfer, vol. 1, pp. 131-138, 1974.

Linne, A., Paciaroni, M., Hall, T., and Parker, T., Ballistic imaging of the near field in a diesel spray, Experiments in fluids, vol. 40, pp. 836-846, 2006.

Lucchini, T., D'Errico, G., and Ettorre, D., Numerical investigation of the spray-mesh-turbulence interactions for highpressure, evaporating sprays at engine conditions, International Journal of Heat and Fluid Flow, vol. 32, pp. 285-297, 2011.

Nordin, N., Complex chemistry modeling of diesel spray combustion, PhD thesis, Chalmers University of Technology, 2001.

OpenFOAM website, The OpenFOAM Foundation, December, 2013.

URL http: / / www . openfoam.org/

Patterson, M. A. and Reitz, R., Modeling the effects of fuel spray characteristics on Diesel engine combustion and emission, SAE Technical Paper 980131, 1998.

Post, S., Iyer, V., and Abraham, J., A study of the near-field entrainment in gas jets and sprays under diesel conditions, ASME Journals of Fluids Engineering, vol. 122, pp. 385$395,2000$.

Powell, C. F., Yue, Y., Poola, R., Wang, J., Lai, M. C., and Shaller, J., X-ray measurements of high pressure fuel sprays, SAE Technical Paper 2001-01-0531, 2001.

Reitz, R. D., Modeling atomization processes in high-pressure vaporizing sprays, Atomization and Spray Technology, vol. 3, pp. 309-337, 1987.
Sandia ECN website, Engine Combustion Network, November, 2013.

URL http: / / www. sandia.gov/ecn/

Schmidt, D. P. and Rutland, C. J., A new droplet collision algorithm, Journal of Computational Physics, vol. 164, pp. 6280, 2000.

Stiesch, G., Modeling engine spray and combustion processes, Springer, 2003.

Taylor, G. I., The instability of liquid surfaces when accelerated in a direction perpendicular to their planes, Batchelor $G K$, The scientific paper of G. I. Taylor, vol. 3, pp. 532-536, 1963.

Vahl, G. D. and Mallinson, G., False diffusion in numerical fluid mechanics. Report 1972/FM/1, University of New South Wales, School of Mechanical and Industrial Engineering, 1972. 